\title{
Evaluation of in-vitro antibiotic susceptibility of different morphological forms of Borrelia burgdorferi
}

\section{Eva Sapi ${ }^{\prime}$}

Navroop Kaur'

Samuel Anyanwu'

David F Luecke'

Akshita Datar'

Seema Patel'

Michael Rossi'

Raphael B Stricker ${ }^{2}$

'Lyme Disease Research Group, Department of Biology and Environmental Sciences, University of New Haven, New Haven, CT, USA; ${ }^{2}$ International Lyme and Associated Diseases Society, Bethesda, MD, USA
This article was published in the following Dove Press journal:

Infection and Drug Resistance

3 May 2011

Number of times this article has been viewed

Background: Lyme disease is a tick-borne illness caused by the spirochete Borrelia burgdorferi. Although antibiotic therapy is usually effective early in the disease, relapse may occur when administration of antibiotics is discontinued. Studies have suggested that resistance and recurrence of Lyme disease might be due to formation of different morphological forms of B. burgdorferi, namely round bodies (cysts) and biofilm-like colonies. Better understanding of the effect of antibiotics on all morphological forms of B. burgdorferi is therefore crucial to provide effective therapy for Lyme disease.

Methods: Three morphological forms of B. burgdorferi (spirochetes, round bodies, and biofilmlike colonies) were generated using novel culture methods. Minimum inhibitory concentration and minimum bactericidal concentration of five antimicrobial agents (doxycycline, amoxicillin, tigecycline, metronidazole, and tinidazole) against spirochetal forms of B. burgdorferi were evaluated using the standard published microdilution technique. The susceptibility of spirochetal and round body forms to the antibiotics was then tested using fluorescent microscopy (BacLight ${ }^{\mathrm{TM}}$ viability staining) and dark field microscopy (direct cell counting), and these results were compared with the microdilution technique. Qualitative and quantitative effects of the antibiotics against biofilm-like colonies were assessed using fluorescent microscopy and dark field microscopy, respectively.

Results: Doxycycline reduced spirochetal structures $\sim 90 \%$ but increased the number of round body forms about twofold. Amoxicillin reduced spirochetal forms by $\sim 85 \%-90 \%$ and round body forms by $\sim 68 \%$, while treatment with metronidazole led to reduction of spirochetal structures by $\sim 90 \%$ and round body forms by $\sim 80 \%$. Tigecycline and tinidazole treatment reduced both spirochetal and round body forms by $\sim 80 \%-90 \%$. When quantitative effects on biofilmlike colonies were evaluated, the five antibiotics reduced formation of these colonies by only $30 \%-55 \%$. In terms of qualitative effects, only tinidazole reduced viable organisms by $\sim 90 \%$. Following treatment with the other antibiotics, viable organisms were detected in $70 \%-85 \%$ of the biofilm-like colonies.

Conclusion: Antibiotics have varying effects on the different morphological forms of B. burgdorferi. Persistence of viable organisms in round body forms and biofilm-like colonies may explain treatment failure and persistent symptoms following antibiotic therapy of Lyme disease.

Keywords: Lyme disease, spirochetes, cysts, round bodies, biofilms

\section{Introduction}

450 Sutter Street, Suite 1504,

San Francisco, CA 94108, USA

Tel +l 4I5 3991035

Fax +I 4153991057

Email rstricker@usmamed.com
Lyme disease is a tickborne illness that was originally described in Old Lyme, Connecticut, in 1975 and subsequently shown to be caused by the spirochete Borrelia burgdorferi. ${ }^{1-4}$ The disease is transmitted by tick vectors of the genus Ixodes. ${ }^{2-4}$ The life cycle and distribution 
of these tick vectors involves rodents, reptiles, birds, and deer. ${ }^{2-4}$ Lyme disease sometimes begins with a skin rash called erythema migrans following a tick bite. The rash may be followed a few weeks or months later by fatigue, musculoskeletal symptoms, neurologic problems, and/or cardiac abnormalities. ${ }^{1-4}$

Over the last 10 years, Lyme disease has grown into a major public health problem in the USA and central Europe..$^{5-7}$ The disease occurs in all age groups with equal prevalence in men and women. ${ }^{5-7}$ In the United States, the Centers for Disease Control and Prevention (CDC) reported that the number of Lyme disease cases has doubled during the last 15 years. ${ }^{5}$ In 2008, a total of 3896 cases of Lyme borreliosis were reported in Connecticut. ${ }^{5}$ However, these figures do not reflect the true incidence of Lyme disease because in 2003 Connecticut stopped requiring mandatory laboratory reporting of the disease. ${ }^{5}$ Therefore, the true number of Lyme disease cases may be at least 10 -fold higher than reported. ${ }^{5-7}$ The increasing trend of the disease has been ascribed to ineffective preventive measures, suboptimal treatment regimens, and incomplete understanding of the nature of the causative spirochete.

The frontline treatment for Lyme disease is administration of antibiotics such as doxycycline, minocycline, amoxicillin, cefuroxime, and ceftriaxone. ${ }^{8-14}$ Although treatment of early Lyme disease is generally successful, studies have shown that in spite of short-course antibiotic therapy of 2-4 weeks, some patients are not successfully treated and go on to develop persistent Lyme disease symptoms. ${ }^{8-11}$ Also, in the absence of sufficient antibiotic treatment in animals and humans, relapse of the disease may occur, suggesting that even after antibiotic treatment, the host immune system fails to prevent recurrence. ${ }^{12-14}$ A possible explanation for this clinical observation is the presence of different morphological forms of B. burgdorferi with differences in sensitivity to the antibiotic treatment. ${ }^{15-30}$

In this study, we developed novel evaluation methods involving optimal culture conditions for three different forms of B. burgdorferi (spirochetes, round bodies, and biofilm-like colonies) and improved bacterial viability determination techniques. These techniques were used to test the effectiveness of antibiotics commonly used for Lyme disease treatment against the different forms of B. burgdorferi. Our goal was to establish a useful in-vitro system to mimic the in-vivo effects of antibiotics on B. burgdorferi in order to develop better therapeutic approaches for Lyme disease.

\section{Materials and methods} Culturing B. burgdorferi

Low passage isolates of the B31 and S297 strains of B. burgdorferi were obtained from the American Type
Culture Collection, Manassas, VA. B. burgdorferi was cultured in Barbour-Stoner-Kelly H (BSK-H) complete medium, with 6\% rabbit serum (Sigma, St Louis, MO, \#B8291). The cultures were incubated at $33^{\circ} \mathrm{C}$ with $5 \%$ $\mathrm{CO}_{2}$ and maintained in sterile $15 \mathrm{~mL}$ glass tubes without antibiotics. Homogeneous cultures having only one form (spirochete) of B. burgdorferi were obtained by maintaining the cultures in a shaking incubator at $33^{\circ} \mathrm{C}$ and $250 \mathrm{rpm}$. At $250 \mathrm{rpm}$, there is no biofilm formation, and the culture remains homogeneous (E Sapi, unpublished observation).

The methods for generation and detection of round body forms of B. burgdorferi using culture tubes and dark field or fluorescent microscopy are described below. For generation of biofilm-like colonies of B. burgdorferi, spirochetes were inoculated in four-well chambers (BD BioCoat ${ }^{\mathrm{TM}}$ CultureSlides, BD Biosciences, Sparks, MD, \#354557) or 24-well plates (BD BioCoat $^{\text {TM }}$ Multiwell Plates, BD Biosciences, Sparks, MD, \#354408) coated with rat-tail collagen type I and incubated for 1 week without shaking. After the 1-week incubation, biofilm-like colonies were visualized using the qualitative and quantitative methods described below.

\section{In-vitro testing of the antibacterial agents} Standard microdilution technique

To determine the minimum inhibitory concentration (MIC) of the antibiotics tested (the lowest concentration that will inhibit visible growth of $B$. burgdorferi spirochetes after a 72-hour incubation period), a standard microdilution method was used. ${ }^{31-33}$ For this procedure, $1 \times 10^{6}$ spirochetes were inoculated into each well of a 48-well tissue culture microplate containing $1 \mathrm{~mL}$ of BSK-H medium per well. The cultures were then treated with $100 \mu \mathrm{L}$ of each antibiotic diluted in phosphate-buffered saline (PBS). Control cultures were treated with PBS alone, and all experiments were run in triplicate. The well plate was covered with parafilm and placed in the incubator for 72 hours. Cell proliferation was assessed using a bacterial counting chamber (Petroff-Hausser Counter-3902) after the 72-hour incubation.

To determine the minimum bactericidal concentration (MBC) of the antibiotics tested (the minimum concentration beyond which no spirochetes can be subcultured after a 3-week incubation period), wells of a 48-well plate were filled with $1 \mathrm{~mL}$ of BSK-H medium, and $20 \mu \mathrm{L}$ of antibiotic-treated spirochetes were added into each of the wells, in triplicate. The well plate was wrapped with parafilm and placed in the incubator for 3 weeks (21 days). After the incubation period, the plate was removed and observed for motile spirochetes in the culture. 


\section{Dark field microscopy and fluorescent microscopy}

To further test the effect of antibiotics on spirochete and round body forms of $B$. burgdorferi, the antibiotics were added to a set of $2-\mathrm{mL}$ polystyrene culture tubes containing spirochetes at a concentration of $1 \times 10^{6}$ cells $/ \mathrm{mL}$ and incubated at $33^{\circ} \mathrm{C}$ with $5 \% \mathrm{CO}_{2}$. These cultures were incubated for various time periods ( 24 hours, 48 hours, 72 hours, and 3 weeks) and cellular growth was scored. For each of these timepoints, cell proliferation assays were performed by directly counting the different morphological forms of $B$. burgdorferi using a bacterial counting chamber and dark field microscopy. Also, by performing LIVE/DEAD ${ }^{\mathbb{R}}$ BacLight ${ }^{\mathrm{TM}}$ Bacterial Viability Assay (Molecular Probes, Inc, Eugene, OR), the ratio of live (green) and dead (red) B. burgdorferi morphological forms was calculated by counting these forms using a bacterial counting chamber and fluorescent microscopy (see below). For the dark field and fluorescent microscopy experiments, a Nikon Eclipse I-series CF160 microscope was used (kindly donated by Dr Alan MacDonald and Turn the Corner Foundation).

\section{Qualitative analysis of biofilm-like colonies}

To qualitatively determine the effect of antibiotics on biofilmlike colonies of $B$. burgdorferi, $1 \times 10^{7}$ cells $/ \mathrm{mL}$ from a homogeneous culture of spirochetes were inoculated in a collagen-coated four-well plate and incubated for 1 week. After the 1-week incubation, biofilm-like colonies were seen in the wells. These biofilm-like colonies were treated with various concentrations of antibiotics diluted in PBS. Control wells were treated with PBS alone, and cultures were run in triplicate. Plates were incubated for 72 hours, and wells were fixed with $500 \mu \mathrm{L}$ of cold alcohol-formalin-acetic acid (AFA) for 20 minutes. The wells were then stained with $200 \mu \mathrm{L}$ of $2 \times$ BacLight $^{\mathrm{TM}}$ staining mixture for 15 minutes in the dark. Coverslips were applied using fluorescent mounting media, and pictures were immediately taken of control and treated wells.

\section{Quantitative analysis of biofilm-like colonies after treatment with antibiotics}

To quantify B. burgdorferi biofilm-like colonies after treatment with various antibiotics, collagen-coated 24 -well plates were inoculated with $2 \times 10^{6}$ cells $/ \mathrm{mL}$ from a homogeneous culture of B. burgdorferi. The plates were incubated for 7 days to generate biofilm-like colonies, and then treated with various concentrations of antibiotics diluted in PBS or with PBS alone, as described above. The plates were incubated for 72 hours, and wells were stained with $1 \mathrm{~mL}$ of crystal violet $(0.1 \%)$ for 10 minutes. After $1 \mathrm{~mL}$ of $95 \%$ ethanol was added to extract stain, the biofilm-like colonies were washed twice with PBS and visualized at an optical density of $570 \mathrm{~nm}$ using a BioTek spectrophotometer.

\section{Statistical analysis}

Statistical analysis was performed by two-sample paired $t$-test using NCSS statistical software (NCSS LLC, Kaysville, UT).

\section{Results}

To compare results from different culture techniques, MIC and $\mathrm{MBC}$ values for antibiotic treatments were calculated and compared with published data (Table 1). The standard published microdilution method involves culturing B. burgdorferi in microwell plates, while the new methodology made use of 2-mL polystyrene test tubes. We observed a significant difference in MIC and MBC values between the two methods. Both MIC and MBC values were in agreement with published data when evaluated by the microdilution protocol. Using the novel culture tube method, MIC values increased $>63$-fold for doxycycline, $>333$-fold for tigecycline, $>333$-fold for amoxicillin, $>833$-fold for metronidazole, and $>694$-fold for tinidazole, compared with our microdilution values (Table 1). Furthermore, MBC values increased $>8$-fold for doxycycline, $>80$-fold for tigecycline, $>40$-fold for amoxicillin, $>50$-fold for metronidazole, and $>25$-fold for tinidazole, compared with our microdilution values (Table 1). Since these MIC and MBC results suggested that our novel method used more optimal culture conditions that allowed the organisms to resist antibiotic treatment, we based further experiments on this method.

To evaluate in-vitro antibiotic sensitivity of spirochete and round body morphological forms, two strains of B. burgdorferi (B31 and S297) were incubated for 72 hours with different antibiotics at concentrations above the calculated MIC and MBC. Antibiotic sensitivity was evaluated using the direct cell counting and dark field morphological evaluation methods (Figure 1). Treatment with these higher concentrations showed that doxycycline reduced spirochetal structures $\sim 90 \%$ but increased the number of cystic round body forms about twofold (Figure 1A). Treatment with metronidazole led to reduction of both spirochetal and cystic round body forms by $\sim 70 \%$ (Figure 1B). Treatment with either tigecycline or tinidazole reduced both spirochetal and cystic round body forms by $\sim 80 \%-90 \%$ (Figures $1 \mathrm{C}$ and $1 \mathrm{D}$ ). Amoxicillin reduced spirochetal structures $\sim 70 \%$ and cystic round body forms by $\sim 68 \%$ (Figure $1 \mathrm{E}$ ). No difference was 
Table I MIC and MBC determination by different methods ${ }^{\mathrm{a}}$

\begin{tabular}{|c|c|c|c|c|c|c|c|c|}
\hline \multirow[t]{2}{*}{ Antibiotics } & \multirow{2}{*}{$\begin{array}{l}\text { Microdilution } \\
\text { method/literature } \\
\text { data }(M I C) \mu \mathrm{g} / \mathrm{mL}\end{array}$} & \multicolumn{3}{|c|}{ Our data (MIC) $\mu \mathrm{g} / \mathrm{mL}$} & \multirow{2}{*}{$\begin{array}{l}\text { Microdilution } \\
\text { method/literature } \\
\text { data }(\mathrm{MBC}) \mu \mathrm{g} / \mathrm{mL}\end{array}$} & \multicolumn{3}{|c|}{ Our data (MBC) $\mu \mathrm{g} / \mathrm{mL}$} \\
\hline & & $\begin{array}{l}\text { Microdilution } \\
\text { method }\end{array}$ & $\begin{array}{l}\text { Direct cell } \\
\text { counting }\end{array}$ & $\begin{array}{l}\text { BacLight }{ }^{\mathrm{TM}} \\
\text { staining }\end{array}$ & & $\begin{array}{l}\text { Microdilution } \\
\text { method }\end{array}$ & $\begin{array}{l}\text { Direct cell } \\
\text { counting }\end{array}$ & $\begin{array}{l}\text { BacLight }^{\mathrm{TM}} \\
\text { staining }\end{array}$ \\
\hline Doxycycline & $0.06-2.00$ & 0.4 & $>25$ & $>25$ & $0.25-6.40$ & 25 & $>200$ & $>200$ \\
\hline Tigecycline & 0.006 & 0.015 & $>5$ & $>5$ & 0.05 & 0.125 & $>10$ & $>10$ \\
\hline Amoxicillin & $0.03-2.00$ & 0.3 & $>100$ & $>100$ & $<0.03-32.00$ & 5 & $>200$ & $>200$ \\
\hline Metronidazole & $0.06-32.00$ & 0.3 & $>250$ & $>250$ & $>4$ & 10 & $>500$ & $>500$ \\
\hline Tinidazole & - & 0.09 & $>62.5$ & $>62.5$ & $>128$ & 10 & $>250$ & $>250$ \\
\hline
\end{tabular}

Notes: ${ }^{\mathrm{C}}$ Comparison of MIC and MBC values for different antibiotics by standard microdilution method (published literature and our data) and novel direct cell counting and fluorescent BacLight ${ }^{\mathrm{TM}}$ staining methods in reference to spirochete forms of Borrelia burgdorferi B3I.

Abbreviations: MBC, minimum bactericidal concentration; MIC, minimum inhibitory concentration.

seen in antibiotic sensitivity testing between the two strains of B. burgdorferi (B31 and S297). Using this method, we found that the most effective doses of the antibiotics against the spirochete forms of B. burgdorferi were $250 \mu \mathrm{g} / \mathrm{mL}$ for doxycycline, $250 \mu \mathrm{g} / \mathrm{mL}$ for metronidazole, $20 \mu \mathrm{g} / \mathrm{mL}$ for tigecycline, $500 \mu \mathrm{g} / \mathrm{mL}$ for tinidazole, and $250 \mu \mathrm{g} / \mathrm{mL}$ for amoxicillin.

To examine in-vitro persistence of spirochete and round body forms of B. burgdorferi, the B31 and S297 strains were incubated for 72 hours and then evaluated by the direct cell counting and dark field evaluation methods (Figure 2). As in the previous set of experiments, doxycycline was found to be more effective against spirochetes while metronidazole and tinidazole were more effective against round body forms (Figure 2A). To test the concept that by discontinuing antibiotics, spirochete forms persist and cystic round body forms convert back to spirochete forms, the treated cultures were sub-cultured in fresh medium without antibiotics. After 3 weeks of subculturing, doxycycline had reduced spirochetal forms by $\sim 45 \%$ and round bodies by $\sim 85 \%$, suggesting that round body forms converted back to spirochete forms in more favorable growth conditions (fresh growth medium and no antibiotic stress). Similarly, metronidazole reduced spirochetal forms by $\sim 50 \%$ and round bodies by $\sim 80 \%$, while tinidazole reduced spirochetal forms by $\sim 94 \%$ and round bodies by $\sim 96 \%$ (Figure $2 B$ ).

To confirm preliminary results, the B31 and S297 strains of B. burgdorferi were further evaluated in vitro for antibiotic sensitivity of spirochete and round body morphological forms by a fluorescent microscopy technique (BacLight ${ }^{\mathrm{TM}}$ staining). B. burgdorferi was incubated for 72 hours with antibiotics at concentrations higher than their calculated MIC and MBC. In this set of experiments, we calculated the post-treatment ratio of live/dead spirochetes and round bodies using $\mathrm{SYTO}^{\circledR} 9$ green-fluorescent nucleic acid stain (live cells) and propidium iodide red-fluorescent nucleic acid stain (dead cells) (Figure 3A). Doxycycline treatment reduced spirochetes by $\sim 94 \%$ but in the remaining
$6 \%$ of the population, $\sim 5 \%$ were still alive (stained green) while $\sim 1 \%$ were dead (stained red). Tinidazole treatment reduced spirochetes by $\sim 95 \%$, but in the remaining $5 \%$ of the population $\sim 3 \%$ were still alive while $\sim 2 \%$ were dead. Tigecycline treatment was most effective as this reduced spirochetes by $\sim 98 \%$, and in the remaining $2 \%$ of the population $\sim 1.5 \%$ were still alive and $\sim 0.5 \%$ were dead. Metronidazole treatment reduced spirochetes by $\sim 54 \%$, but in the remaining $46 \%$ of the population $\sim 45 \%$ were still alive and only $\sim 1 \%$ were dead. Amoxicillin treatment reduced spirochetes by $\sim 69 \%$, but in the remaining $31 \%$ of the population $\sim 30 \%$ were alive and only $\sim 1 \%$ cells were dead (Figure 3A).

Doxycycline treatment increased round bodies by $\sim 275 \%$ (Figure 3B). Out of this population $\sim 270 \%$ were alive and only $\sim 5 \%$ were dead. Tinidazole treatment reduced round bodies by $\sim 94 \%$, but in the remaining $6 \%$ of the population only $\sim 2 \%$ were dead. Tigecycline treatment reduced round bodies by $\sim 96 \%$, but in the remaining $4 \%$ of population only $\sim 1 \%$ were dead. Metronidazole treatment reduced round bodies by $\sim 68 \%$, but in the remaining $32 \%$ of the population $\sim 15 \%$ were dead. Amoxicillin treatment reduced round bodies by $\sim 32 \%$, but in the remaining $68 \%$ of the population $\sim 30 \%$ were dead (Figure 3B). The microscopic appearance of the B31 and S297 strains of B. burgdorferi following treatment with each antibiotic is shown in Figures $3 \mathrm{C}$ and $3 \mathrm{D}$, respectively.

In the next experiments, a biofilm-like form of B. burgdorferi was evaluated quantitatively using a crystal violet staining method and qualitatively using the fluorescent microscopy technique (BacLight ${ }^{\mathrm{TM}}$ staining) (Figure 4). Using the quantitative staining method, doxycycline reduced biofilm-like colonies by $\sim 40 \%$, tinidazole reduced biofilmlike colonies by $\sim 50 \%-55 \%$, tigecycline reduced biofilm-like colonies by $\sim 35 \%$, and amoxicillin and metronidazole reduced biofilm-like colonies by $\sim 30 \%$ (Figure $4 \mathrm{~A}$ ). For qualitative 
A

Effect of doxycycline at different concentrations on

B. burgdorferi-B31 strain after 72 hours

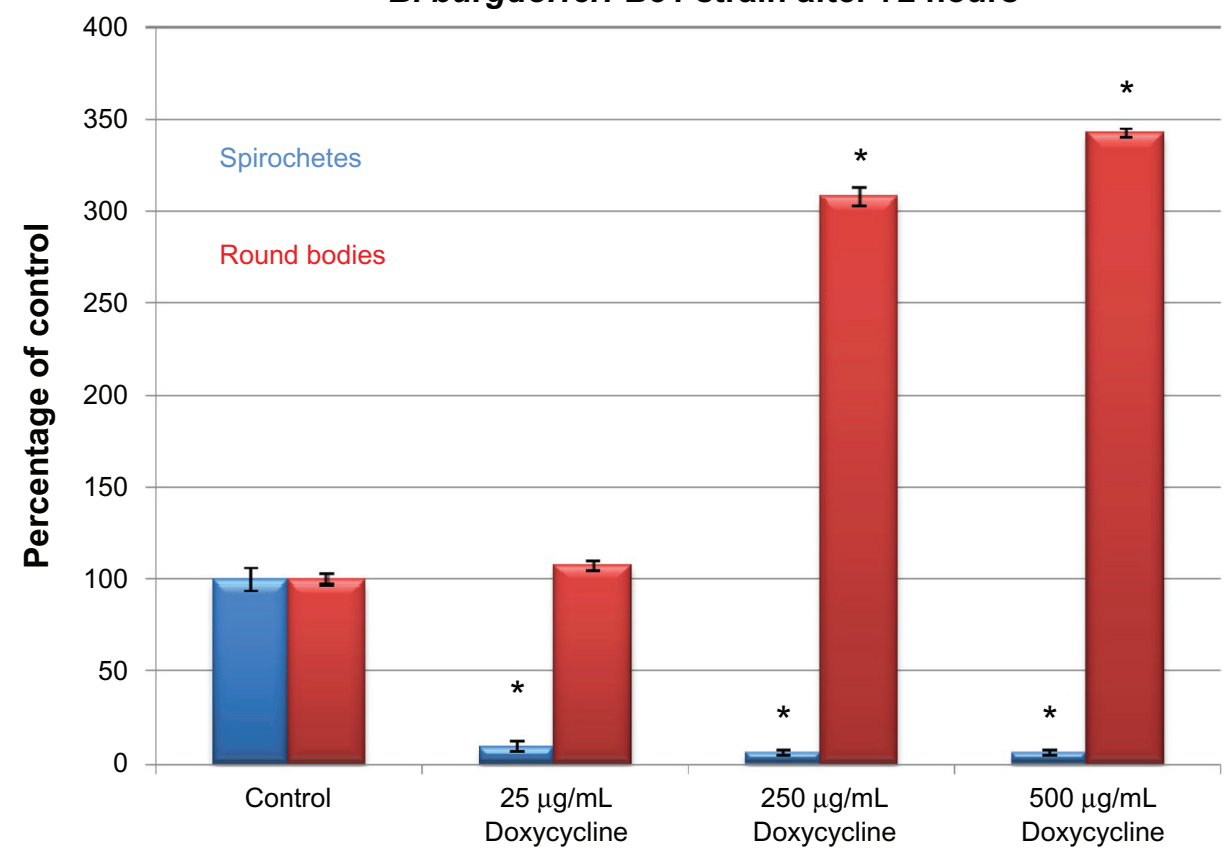

B

Effect of doxycycline at different concentrations on

B. burgdorferi-S297 strain after 72 hours

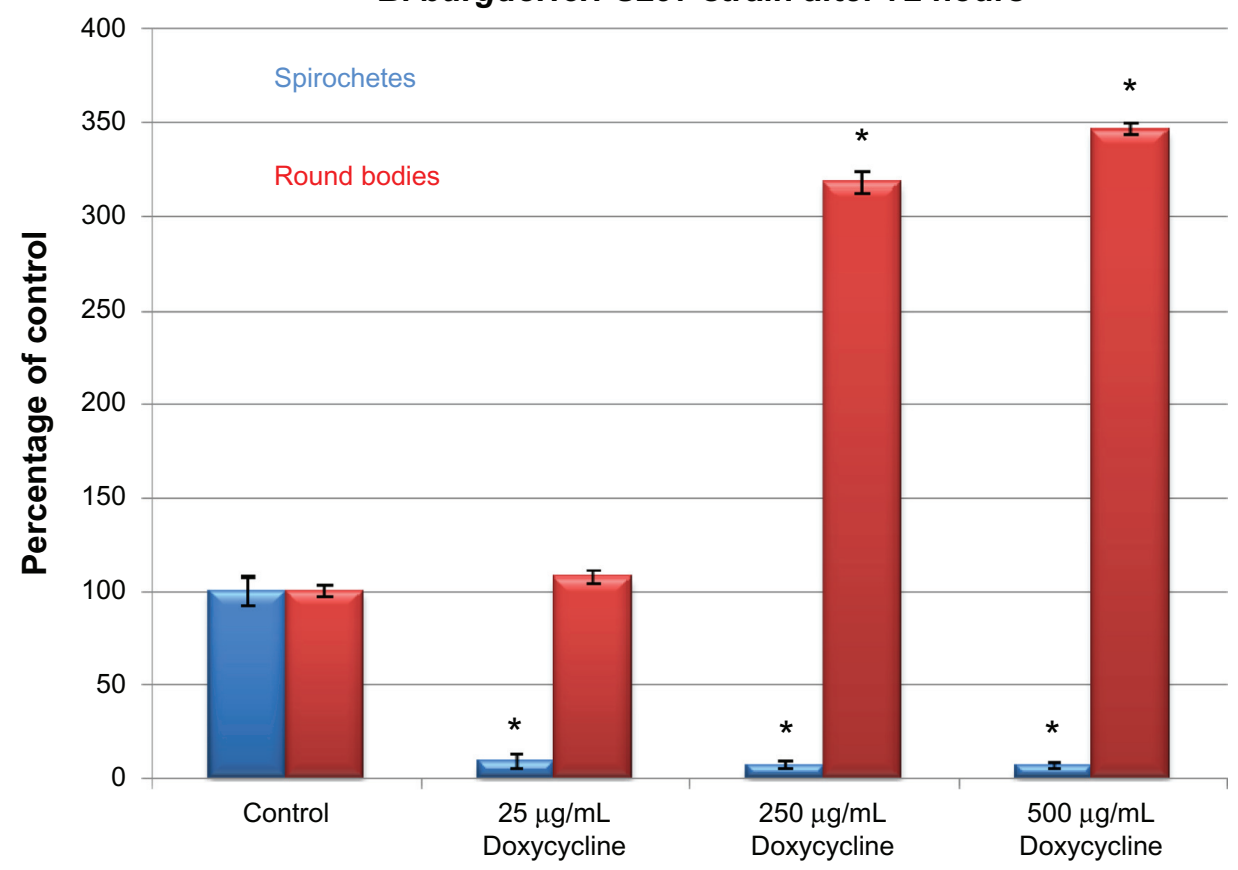

Figure IA Susceptibility of the spirochete and round body forms of strain B3I (top panels) and strain S297 (bottom panels) of B. burgdorferi to different concentrations (between calculated MIC and MBC) of five antibiotics after 72-hour treatment measured by dark-field microscopy.

Note: $* P$ values $<0.05$ indicates statistical significance compared with control.

analysis of bacterial cells in biofilm-like colonies, cultures were treated as described above for 72 hours and stained with BacLight ${ }^{\mathrm{TM}}$ fluorescent viability stain (Figure 4B). In the absence of antimicrobial agents, B. burgdorferi form biofilm-like colonies in which $\sim 98 \%$ of the colonies stain green (live cells) and $\sim 2 \%$ red (dead cells) (Figure $4 \mathrm{Ba}$ ). Doxycycline-treated colonies were similar in size to control colonies and $\sim 70 \%$ stained green and $\sim 30 \%$ stained red (Figure $4 \mathrm{Bb}$ ). Tinidazole-treated colonies were very tiny and loose in their morphology, and $\sim 10 \%$ stained green and $\sim 90 \%$ 


\section{A}

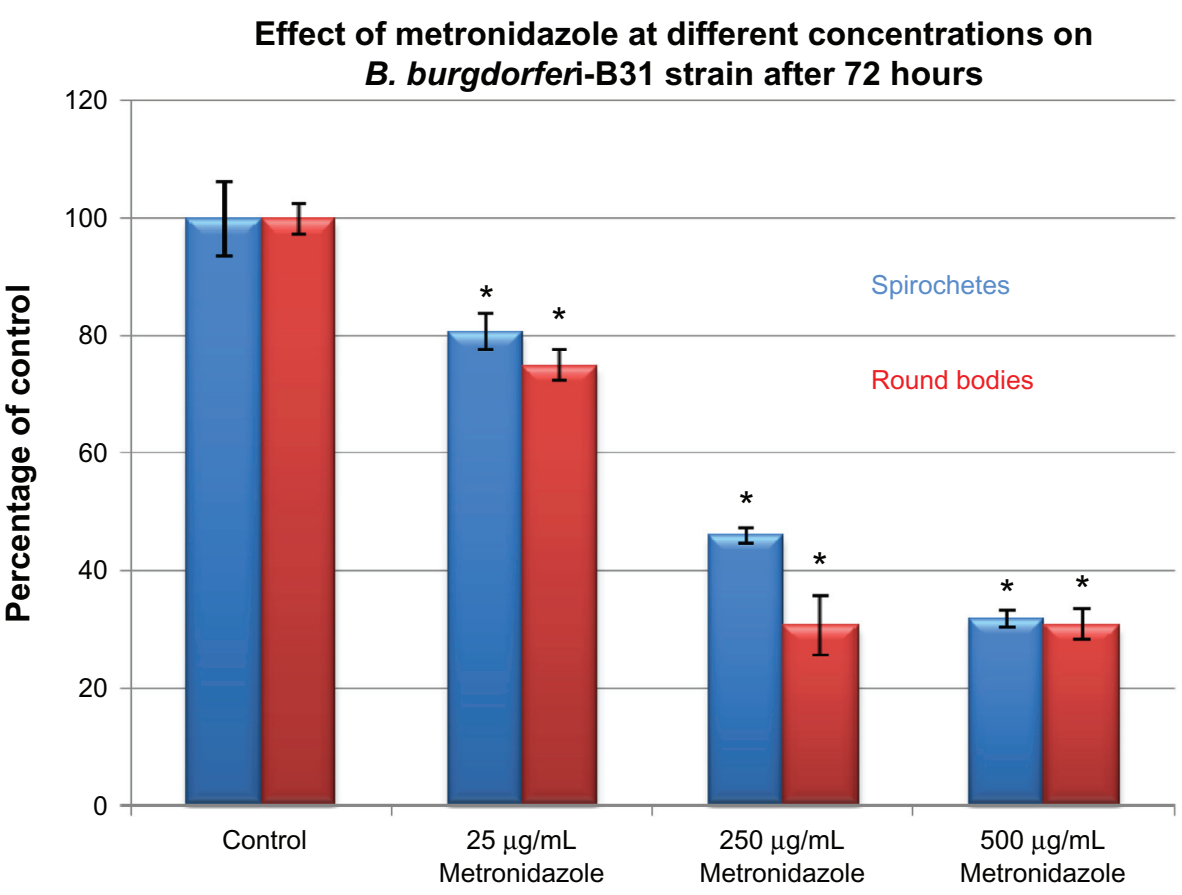

B

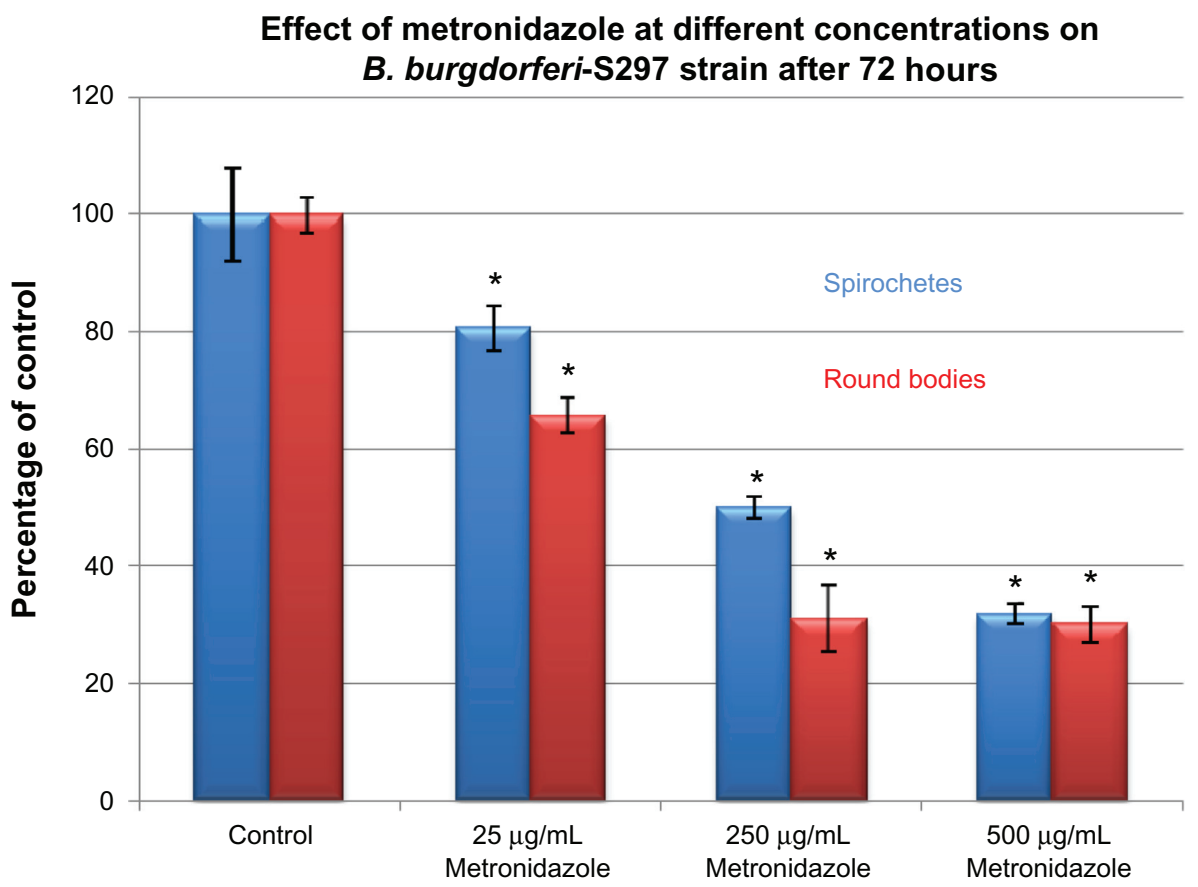

Figure IB Susceptibility of the spirochete and round body forms of strain B3I (top panels) and strain S297 (bottom panels) of B. burgdorferi to different concentrations (between calculated MIC and MBC) of five antibiotics after 72-hour treatment measured by dark-field microscopy.

Note: $* P$ values $<0.05$ indicates statistical significance compared with control.

stained red (Figure 4Bc). Tigecycline-treated colonies were similar in size to control colonies, and $\sim 70 \%$ stained green and $\sim 30 \%$ stained red (Figure 4Bd). Metronidazole-treated and amoxicillin-treated colonies were larger in size than control colonies, and $\sim 80 \%-85 \%$ stained green while $\sim 20 \%$ stained red (Figure 4Be and 4Bf).

\section{Discussion}

The goal of our study was to demonstrate the in-vitro susceptibility of different morphological forms of $B$. burgdorferi to various antibiotics using improved technical approaches in order to understand why antibiotic treatment for patients with Lyme disease could fail. To successfully eradicate 
A

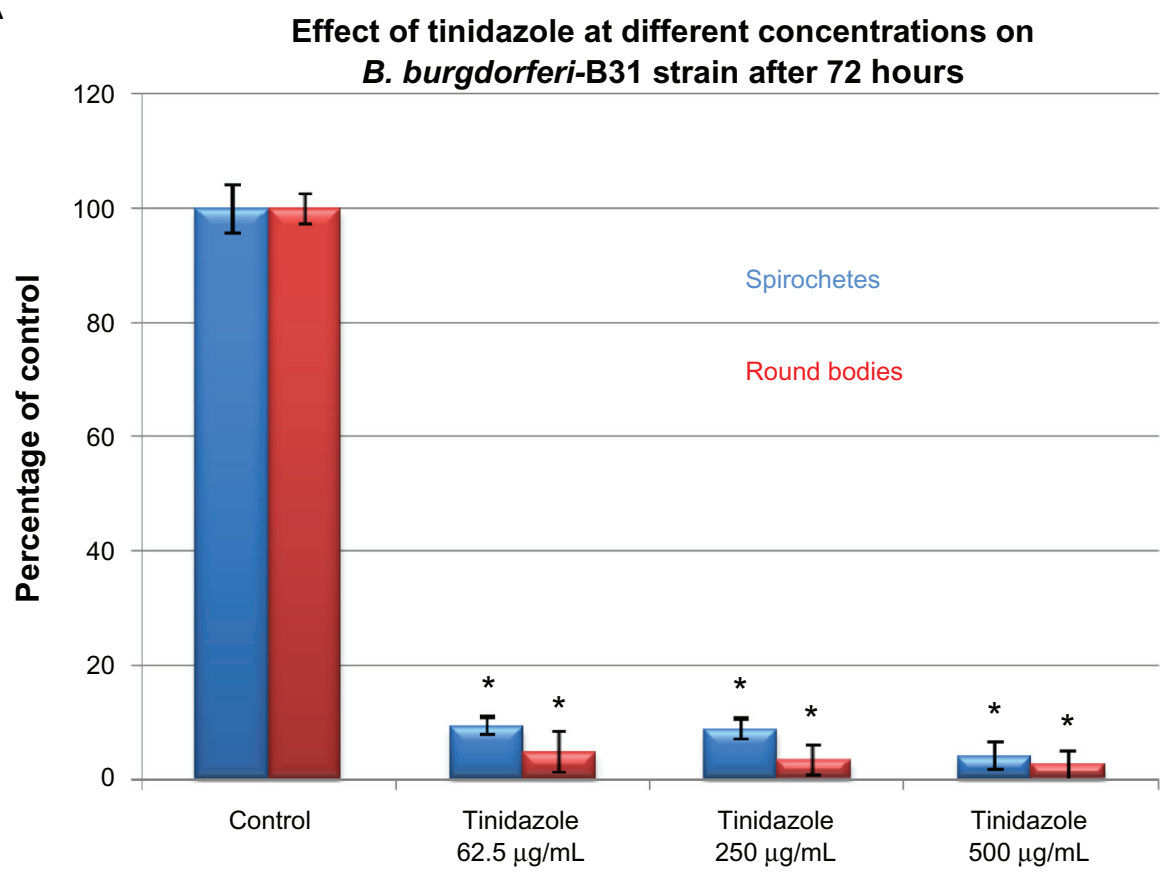

B

Effect of tinidazole at different concentrations on

B. burgdorferi-S297 strain after 72 hours

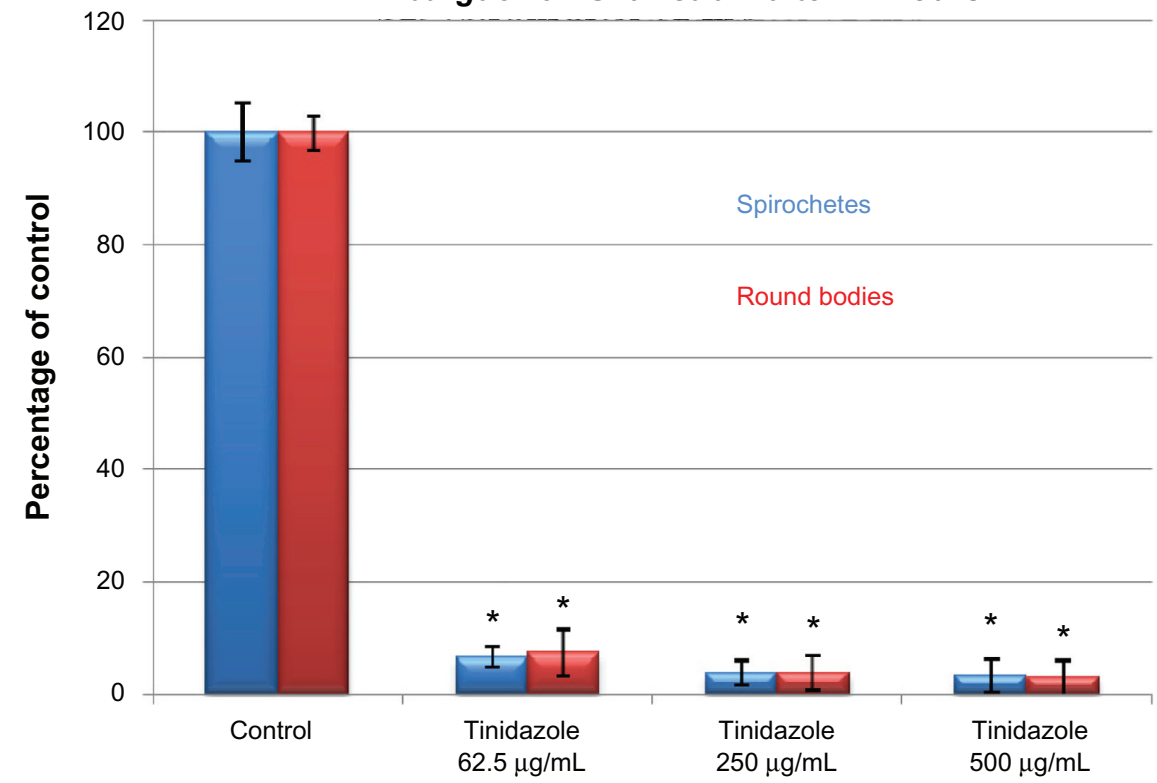

Figure IC Susceptibility of the spirochete and round body forms of strain B3I (top panels) and strain S297 (bottom panels) of B. burgdorferi to different concentrations (between calculated MIC and MBC) of five antibiotics after 72-hour treatment measured by dark-field microscopy.

Note: $* P$ values $<0.05$ indicates statistical significance compared with control.

B. burgdorferi, antimicrobial agents should eliminate all morphological forms of the organism. Furthermore, for better demonstration of antibiotic susceptibility of different morphological forms of B. burgdorferi, there is a need for reliable in-vitro testing methods.

The spirochete form of B. burgdorferi is the most active form, with periplasmic flagella that make the organisms motile. ${ }^{15,16}$ Spirochetes can also enter into tissues and cause intracellular infection. ${ }^{17,18}$ Adverse environmental conditions such as change in temperature, $\mathrm{pH}$, starvation, and most importantly antibiotic exposure can cause a phenotypic change in the spirochete. ${ }^{19,20}$ This change involving surface proteins is hypothesized to be the way in which the spirochete evades the host immune system. 


\section{A}

Effect of tigecycline at different concentrations on

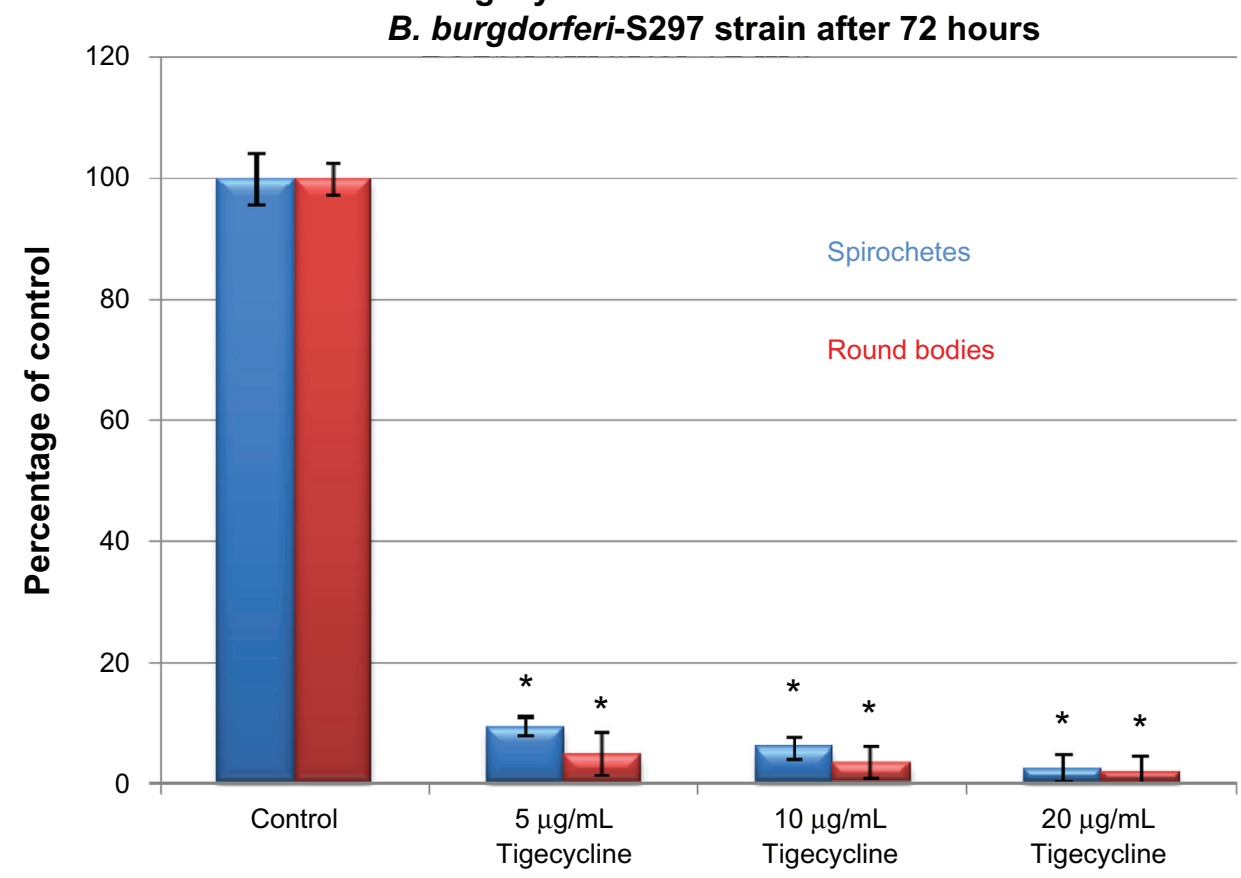

B

Effect of tigecycline at different concentrations on

B. burgdorferi-S297 strain after 72 hours

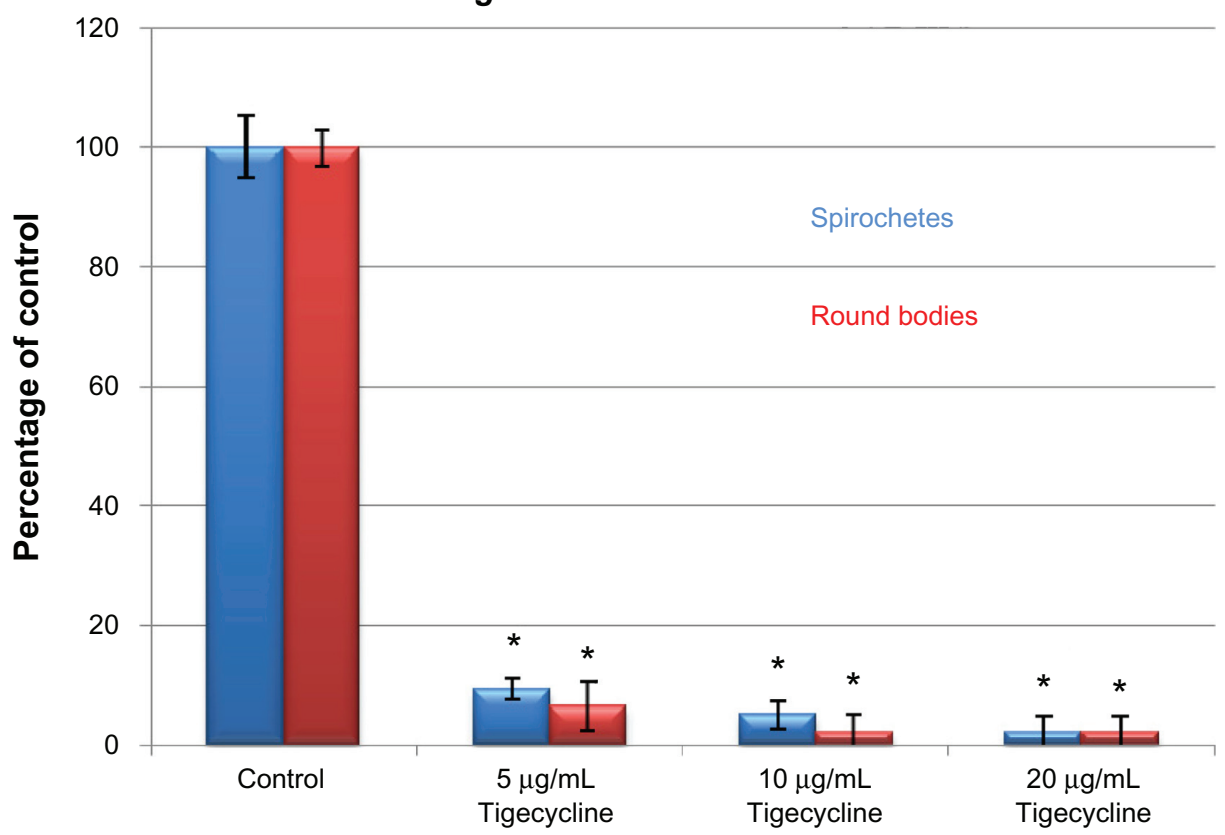

Figure ID Susceptibility of the spirochete and round body forms of strain B3I (top panels) and strain S297 (bottom panels) of B. burgdorferi to different concentrations (between calculated MIC and MBC) of five antibiotics after 72-hour treatment measured by dark-field microscopy.

Note: $* P$ values $<0.05$ indicates statistical significance compared with control.

The change in phenotypic expression could also lead to structural alterations in the spirochete form and induction of the cyst form, a knob-shaped structure containing one or multiple spirochetes. ${ }^{21-24}$ Cysts have a low metabolic rate that enables them to survive in a hostile environment until conditions become favorable for them to multiply again. ${ }^{21-25}$ These cyst forms have been detected in spinal fluid, and have been linked to neuroborreliosis. ${ }^{22}$ The spirochetes can also disintegrate into minute particles called granules. ${ }^{25}$ These granules are liberated through the periplasmic sheath surrounding 
A

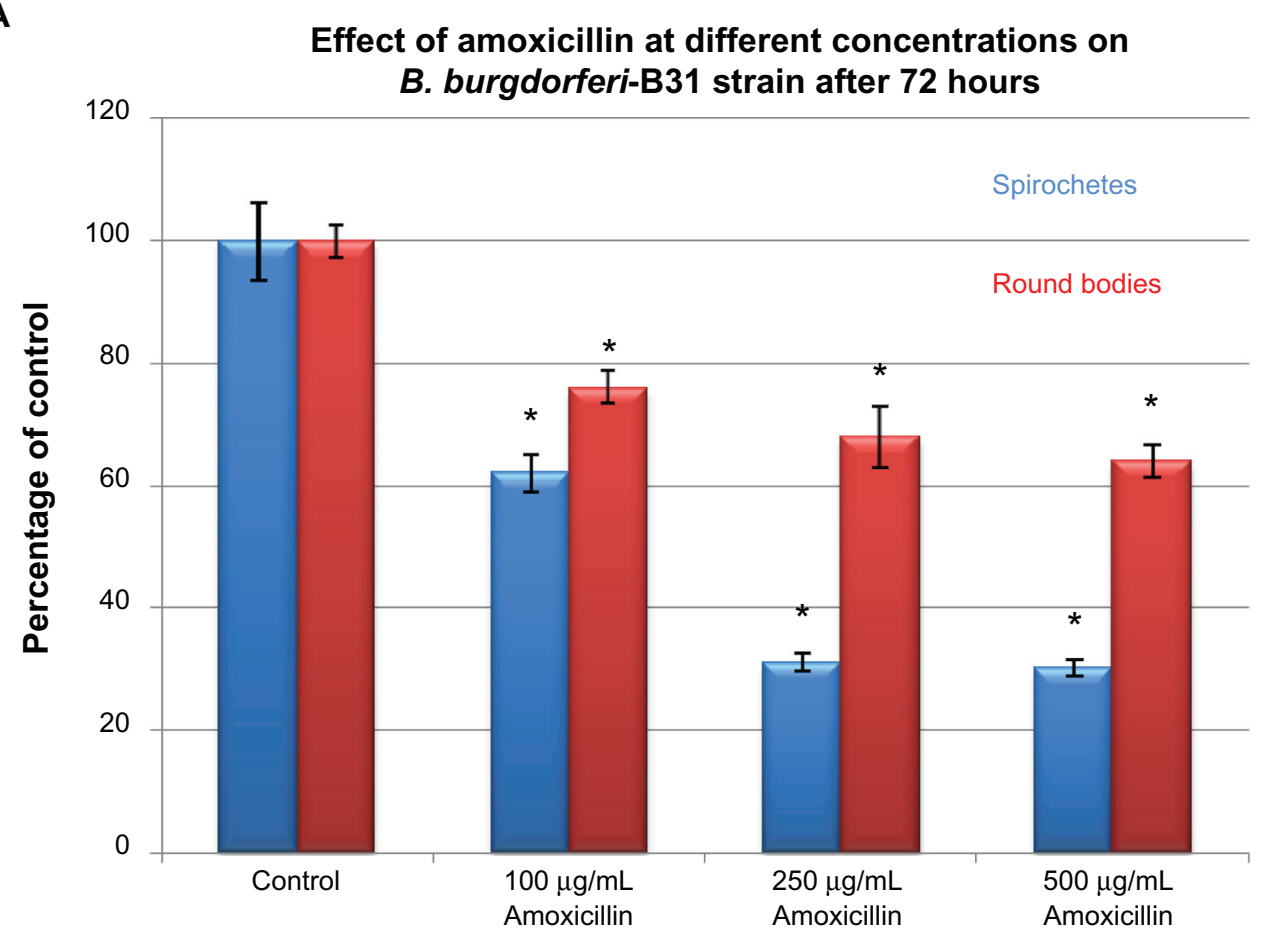

B

Effect of amoxicillin at different concentrations on B. burgdorferi-S297 strain after 72 hours

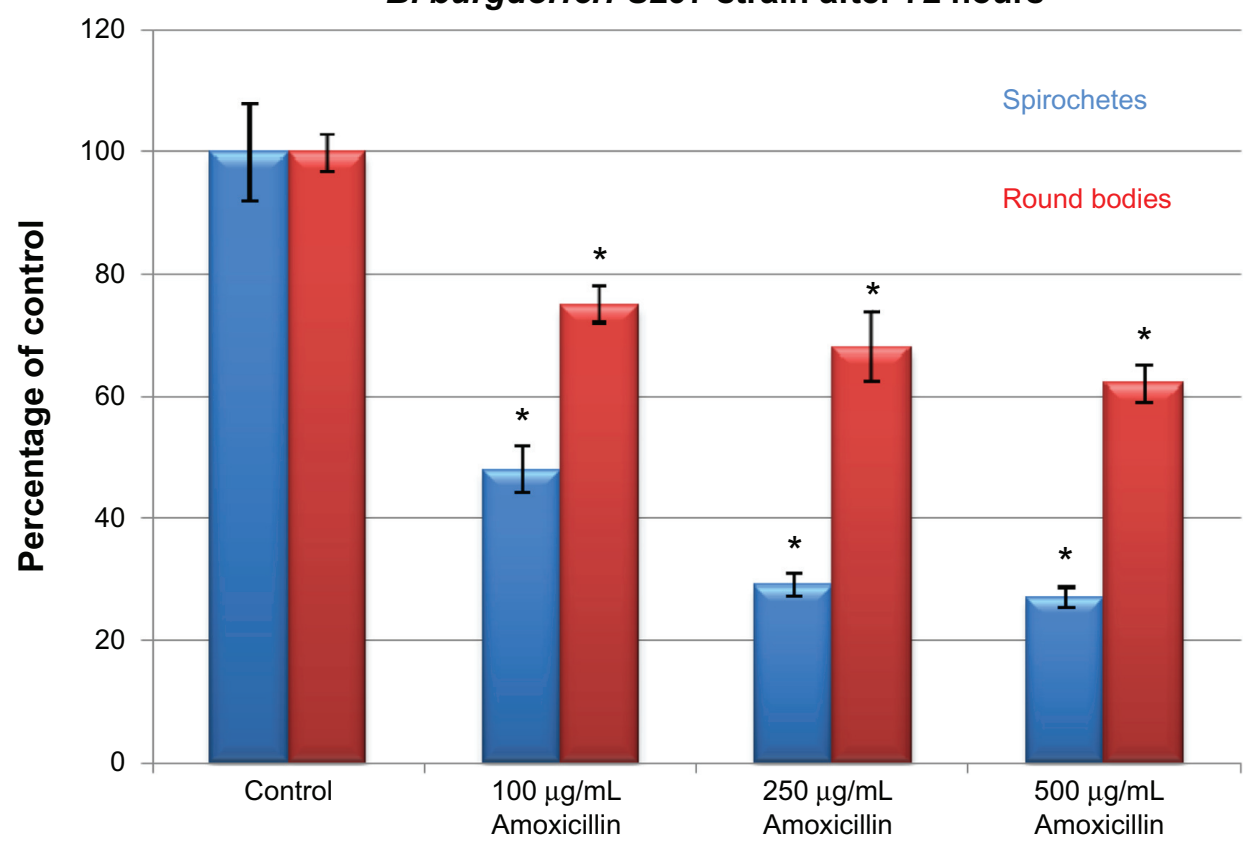

Figure IE Susceptibility of the spirochete and round body forms of strain B3I (top panels) and strain S297 (bottom panels) of B. burgdorferi to different concentrations (between calculated MIC and MBC) of five antibiotics after 72-hour treatment measured by dark-field microscopy.

Note: $* P$ values $<0.05$ indicates statistical significance compared with control.

the spirochete body by budding and extrusion, and they may also be transmissible. ${ }^{25}$ Both cysts and granules are together referred to as round body forms in this study. Several studies have shown that $B$. burgdorferi can convert from the spirochete form to the round body form in vitro when presented with an unfavorable environment, and the organism can revert back to the spirochete form when conditions are again favorable for growth. ${ }^{21-24}$ The presence of atypical forms of $B$. burgdorferi may be the reason why the spirochete can survive in infected tissues for years or even for decades. ${ }^{21-24}$ 
A

Effect of tinidazole, metronidazole and doxycycline at different

concentrations on B. burgdorferi-B31 strain after 72 hours

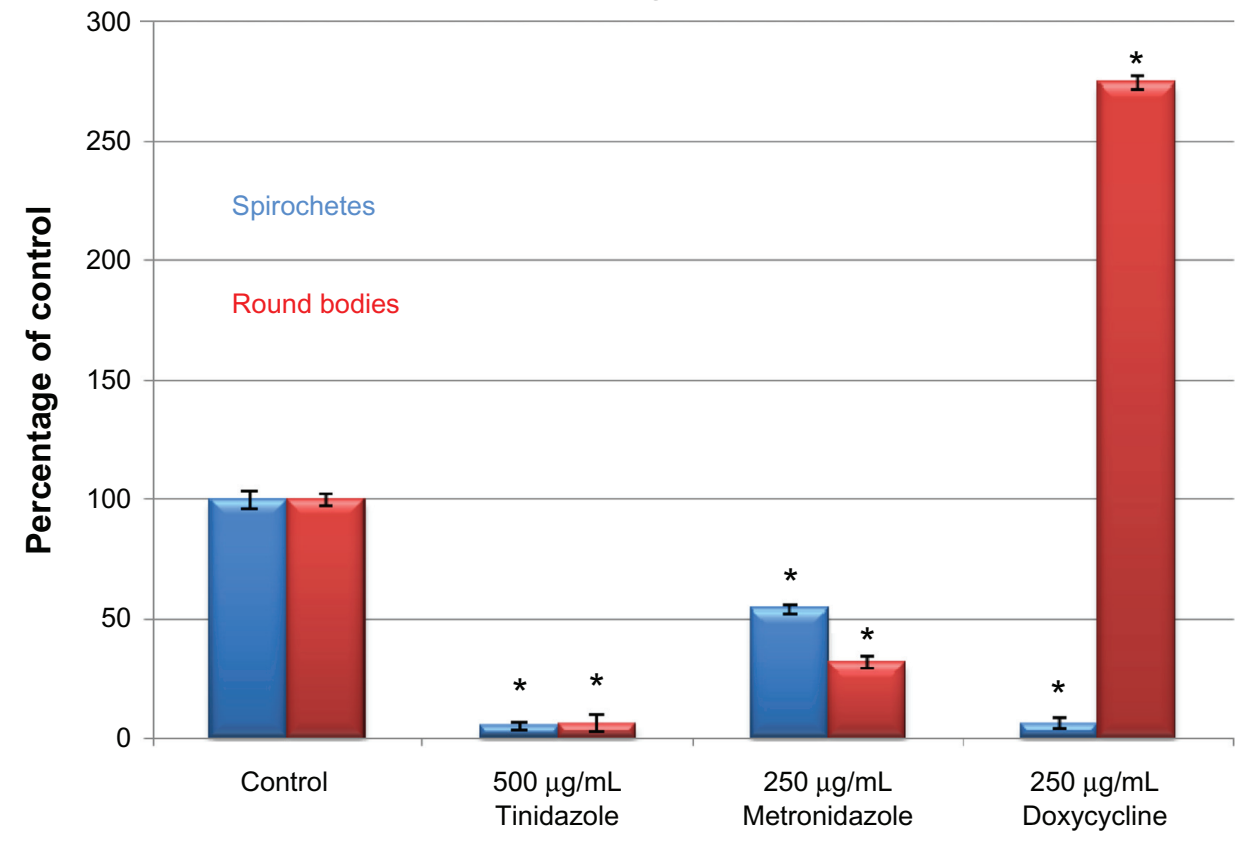

B
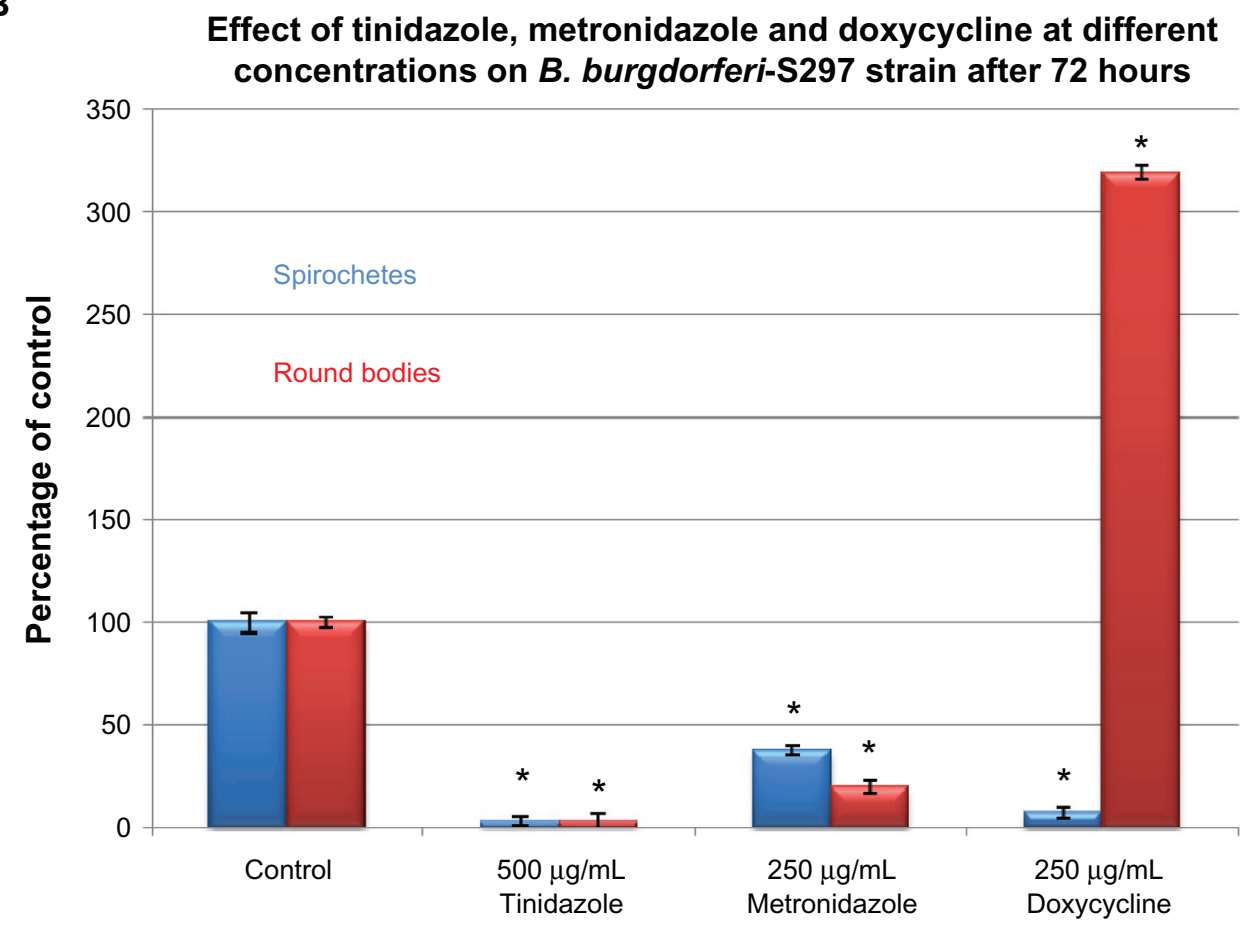

Figure 2A Susceptibility of the spirochete and round body forms of strain B3I (top panels) and strain S297 (bottom panels) of B. burgdorferi to the most effective concentrations of three antibiotics measured by dark-field microscopy. Tinidazole, metronidazole, and doxycycline effect on B. burgdorferi after 72-hour treatment. Note: $* P$ values $<0.05$ indicates statistical significance compared with control.

In addition to round body forms, we and others recently noted that $B$. burgdorfer $i$ has the capability to form organized structures called biofilm-like colonies. ${ }^{26,27}$ Biofilms are adherent polysaccharide-based matrices that protect bacteria from the hostile host environment and facilitate persistent infection. ${ }^{28-30}$ These organized structures are responsible for a number of chronic infections, including periodontitis, chronic otitis media, endocarditis, gastrointestinal infection, and chronic lung infection. Formation of biofilm-like colonies would allow B. burgdorferi to survive various environmental 
A

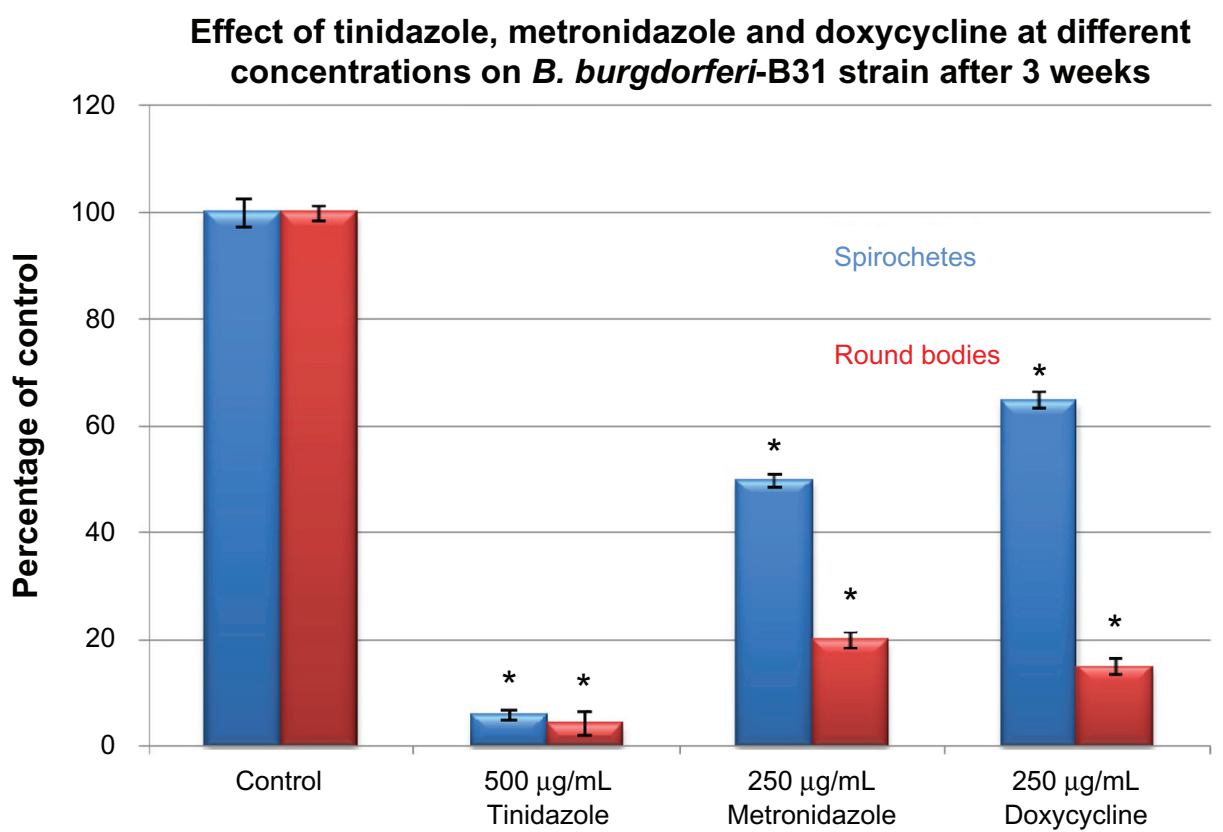

B

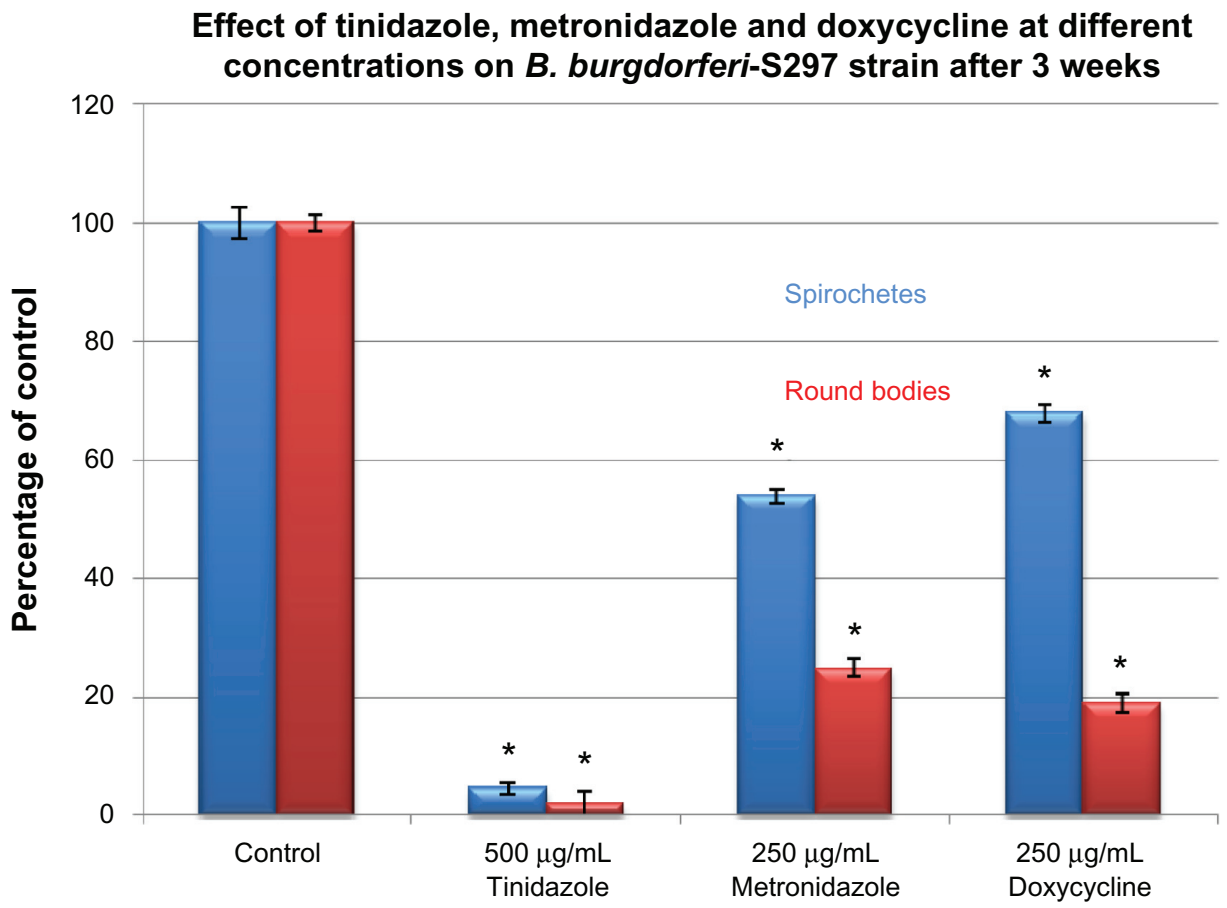

Figure 2B Susceptibility of the spirochete and round body forms of strain B3I (top panels) and strain S297 (bottom panels) of B. burgdorferi to the most effective concentrations of three antibiotics measured by dark-field microscopy. Tinidazole, metronidazole, and doxycycline effect on B. burgdorferi after 3 weeks of subculturing following 72-hour treatment.

Note: $* P$ values $<0.05$ indicates statistical significance compared with control.

stresses including exposure to antibacterial agents. ${ }^{28-30}$ Recent studies suggest that bacteria live in an environment deep within the biofilm-like colonies where diffusion of antibiotics might be difficult, and in that state the bacteria could become 1000 times more resistant to antibiotics. ${ }^{28-30}$ This resistance could also be one of the reasons why conventional antibiotic therapy that is usually effective against free-floating bacteria becomes ineffective once a pathogen forms biofilm-like colonies. ${ }^{28-30}$

In this study, novel methods of in-vitro antibiotic susceptibility evaluation were used. These methods include optimal culture and treatment conditions such as the culture apparatus (tubes, to limit oxygen content), temperature, density 


\section{A}

Percentage live and dead spirochetes after treatment of B31 strain with different antibiotics at their most effective concentration after 72 hours

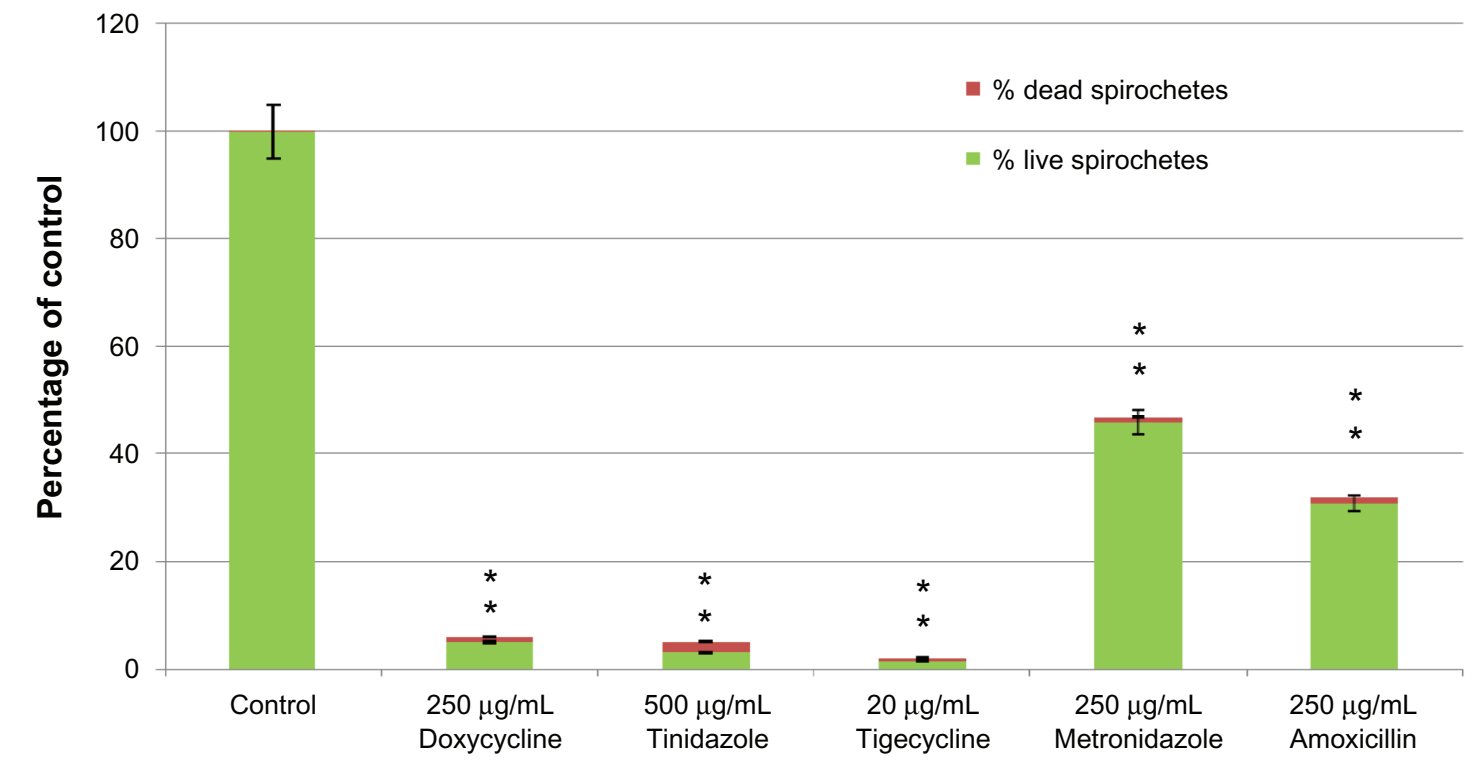

B

Percentage live and dead spirochetes after treatment of S297 strain with different antibiotics at their most effective concentration after 72 hours

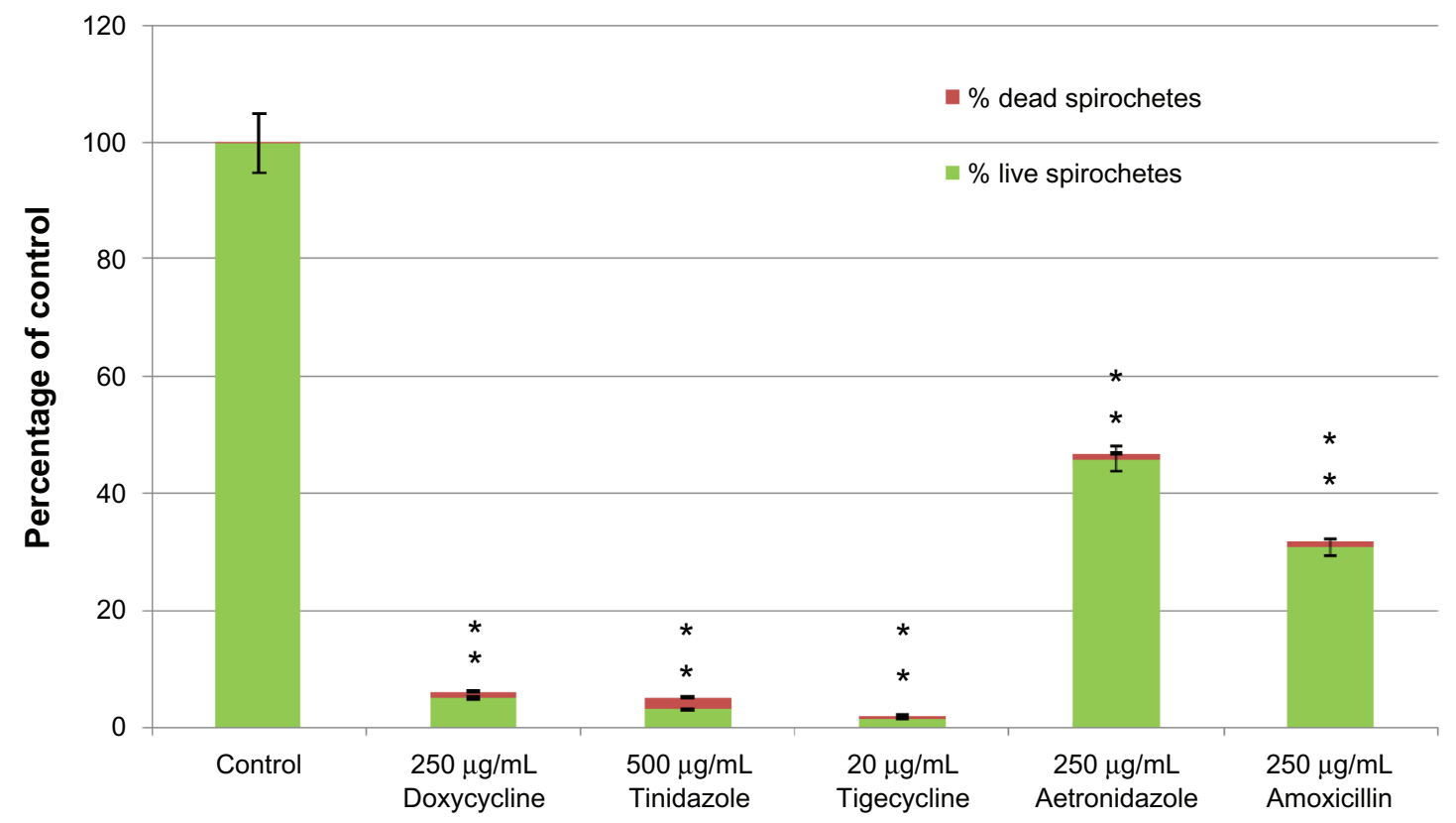

Figure 3A Evaluation of live/dead spirochete and round body forms of B. burgdorferi following treatment with five antibiotics measured by fluorescent microscopy using SYTO ${ }^{\circledR 9}$ green-fluorescent stain (live organisms) and propidium iodide red-fluorescent stain (dead organisms). Effect of doxycycline, tinidazole, tigecycline, metronidazole, and amoxicillin on spirochete forms of strain B3I (top panel) and strain S297 (bottom panel).

Notes: $* P$ values calculated were $<0.05$ indicating statistical significance compared with control for live and dead spirochetes.

of inoculum, amount of culture medium, and $\mathrm{CO}_{2}$ level..$^{34,35}$ These methods should counteract problems with culture variability of $B$. burgdorferi strains that have been described in the past. ${ }^{36,37}$ Furthermore our novel procedures involve better bacterial viability determination methods such as fluorescent and dark field microscopy. These microscopic evaluation methods are more reliable and sensitive than the standard published bacterial viability determination protocols. ${ }^{38,39}$ Being metabolically inactive, round body forms of $B$. burgdorferi could not be detected by standard protocols, but they can be directly visualized under the microscope by our novel evaluation methods. This explains why the standard protocols measure 
A

Percentage live and dead round bodies after treatment of B31 strain with different antibiotics at their most effective concentration after 72 hours

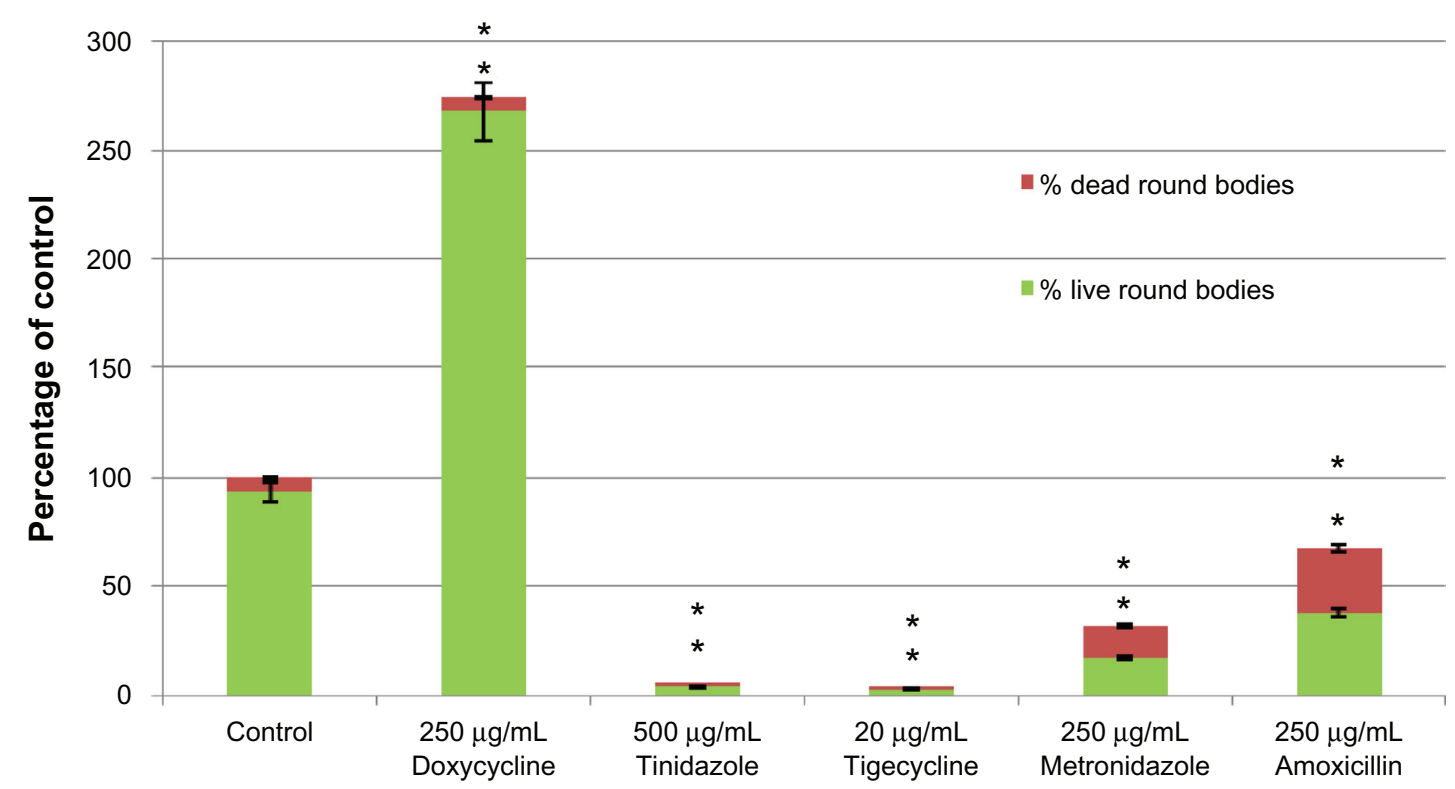

B

\section{Percentage live and dead round bodies after treatment of S297 strain with different} antibiotics at their most effective concentration after 72 hours

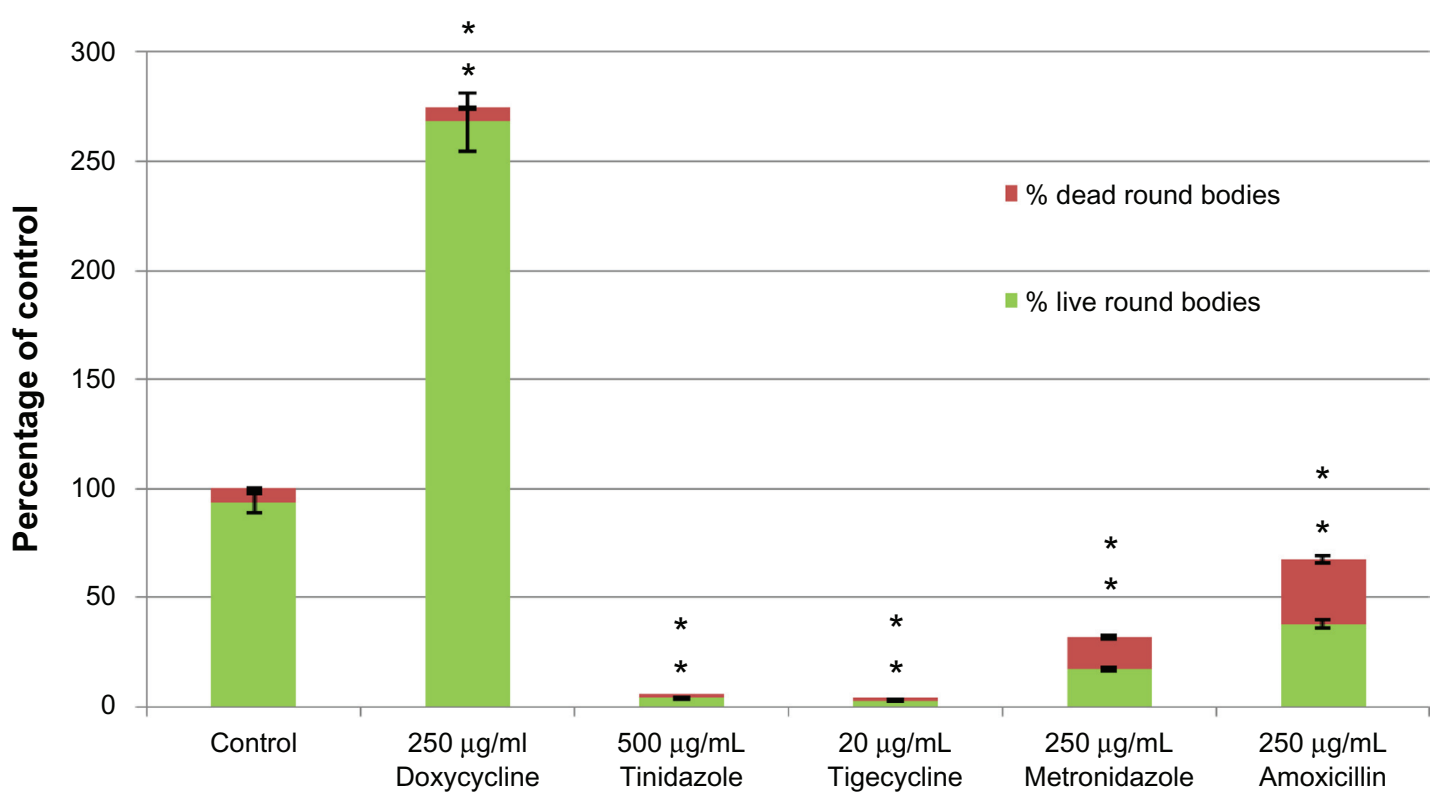

Figure 3B Evaluation of live/dead spirochete and round body forms of B. burgdorferi following treatment with five antibiotics measured by fluorescent microscopy using SYTO ${ }^{\circledR 9}$ green-fluorescent stain (live organisms) and propidium iodide red-fluorescent stain (dead organisms). Effect of doxycycline, tinidazole, tigecycline, metronidazole and amoxicillin on round body forms of strain B3I (top panel) and strain S297 (bottom panel).

Notes: $* P$ values calculated were $<0.05$ indicating statistical significance compared with control for live and dead round bodies.

effectiveness of antibiotics only in reference to metabolically active spirochete forms while our novel antibiotic sensitivity study measures effectiveness of antibiotics in reference to all morphological forms of B. burgdorferi.

We found that doxycycline significantly reduced the spirochete form of B. burgdorferi $(\sim 90 \%)$ but also increased the round body forms twofold. Amoxicillin reduced spirochetal forms by $\sim 85 \%-90 \%$ and round body forms by $\sim 68 \%$. In contrast, metronidazole, tinidazole, and tigecycline significantly decreased both the spirochete and the round body forms of B. burgdorferi, but live organisms could still be detected following treatment 

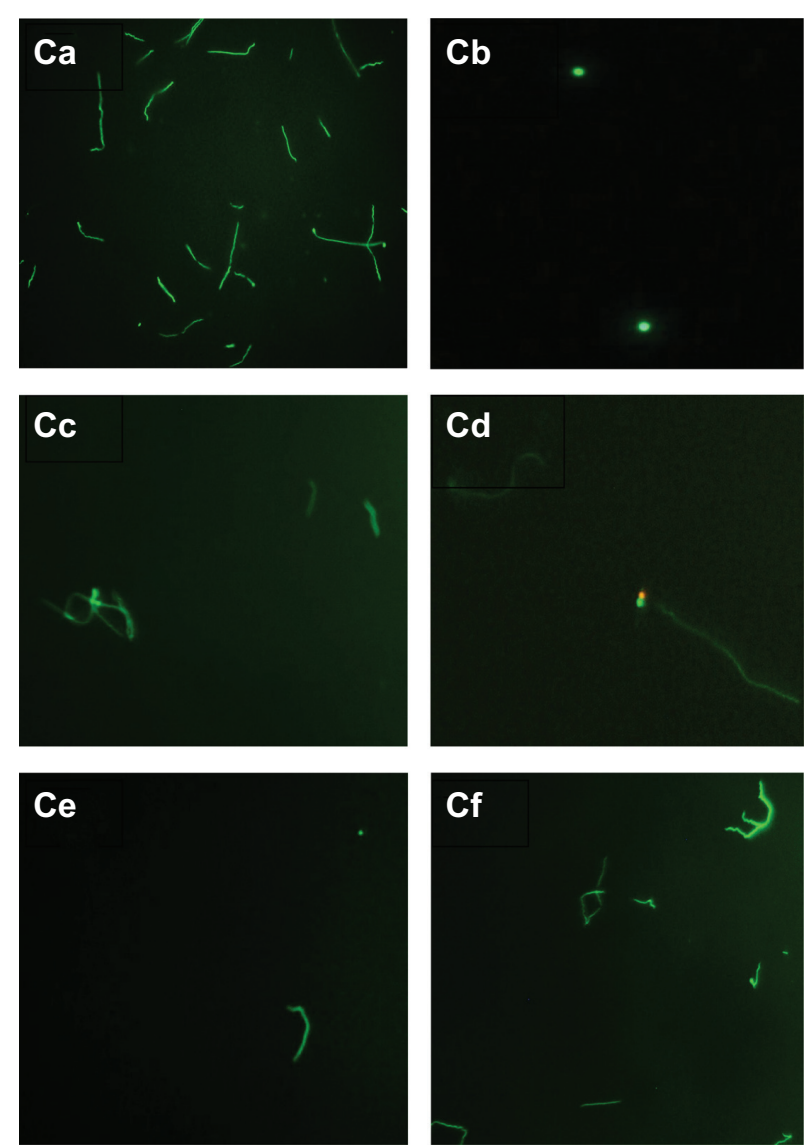

Figure 3C Evaluation of live/dead spirochete and round body forms of B. burgdorferi following treatment with five antibiotics measured by fluorescent microscopy using SYTO ${ }^{\circledR 9}$ green-fluorescent stain (live organisms) and propidium iodide red-fluorescent stain (dead organisms). Visualization of spirochete and round body forms of strain B3I following antibiotic treatment measured by dark field microscopy: $(\mathrm{Ca})$ Control; $(\mathrm{Cb})$ Doxycycline; (Cc) Tinidazole; (Cd) Metronidazole; (Ce) Tigecycline; (Cf) Amoxicillin. Note: All images taken at $40 \times$ magnification.

with these agents. Furthermore, the antibiotics studied were equally effective or ineffective against two different strains (B31 and S297) of B. burgdorferi. This observation confirms the reliability of our experimental technique. It remains to be seen whether combinations of antibiotics would be more effective than individual antibiotics alone in our in-vitro culture system.

Our results delineate antibiotic concentrations that are effective in vitro. Whether equivalent concentrations can be attained with clinical use of these agents in vivo remains to be determined. $.^{39}-45$ An in-vitro study showed that tigecycline destroyed the spirochete and round body forms of B. burgdorferi. ${ }^{39}$ However, an in-vivo study in mice showed that tigecycline was ineffective during the late stage of Lyme disease based on the persistence of viable and infectious but nondividing or slowly dividing organisms in the animals. ${ }^{46}$ Our study demonstrated that tigecycline was effective against the
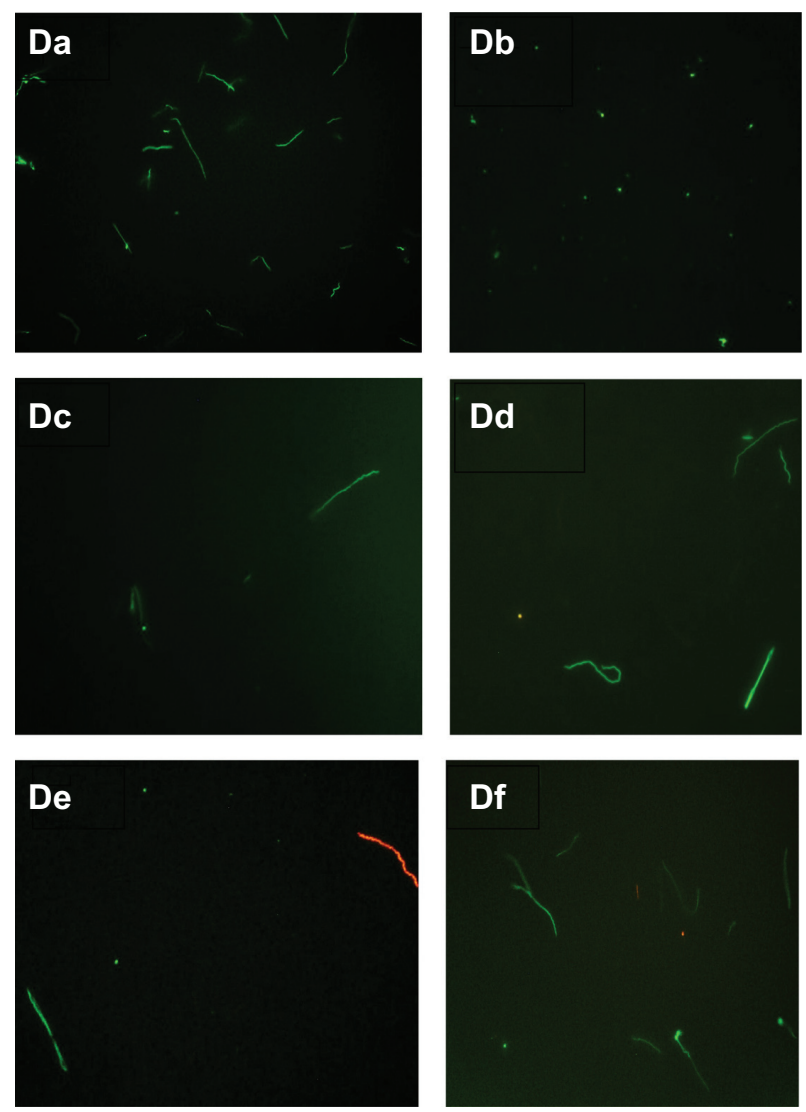

Figure 3D Evaluation of live/dead spirochete and round body forms of B. burgdorferi following treatment with five antibiotics measured by fluorescent microscopy using SYTO ${ }^{\circledR 9}$ green-fluorescent stain (live organisms) and propidium iodide red-fluorescent stain (dead organisms). Visualization of spirochete and round body forms of strain S297 following antibiotic treatment measured by dark field microscopy: (Da) Control; (Db) Doxycycline; (Dc) Tinidazole; (Dd) Metronidazole; (De) Tigecycline; (Df) Amoxicillin.

Note: All images taken at $40 \times$ magnification.

spirochete and round body forms of B. burgdorferi but was not effective against the biofilm-like mass. One possible explanation for the conflicting in-vitro and in-vivo results could be the presence of these biofilm-like colonies in the late stage of the disease, which renders B. burgdorferi more resistant against the antibiotic. Another possibility is that intracellular invasion of the spirochete in vivo could protect it from the action of antibiotics. ${ }^{17,18}$ Further evaluation of $B$. burgdorferi localization in tissues and biofilm-like masses is warranted.

To summarize, this study outlines novel in-vitro methods to determine optimal growth conditions for B. burgdorferi. The study also describes novel microscopic viability determination methods to assess three morphological forms of B. burgdorferi (spirochetes, round bodies, and biofilm-like colonies), and the methods were used to evaluate antibiotic susceptibility of the different morphological forms of this complex organism. Our in-vitro methodology will facilitate the design of experiments 


\section{A}
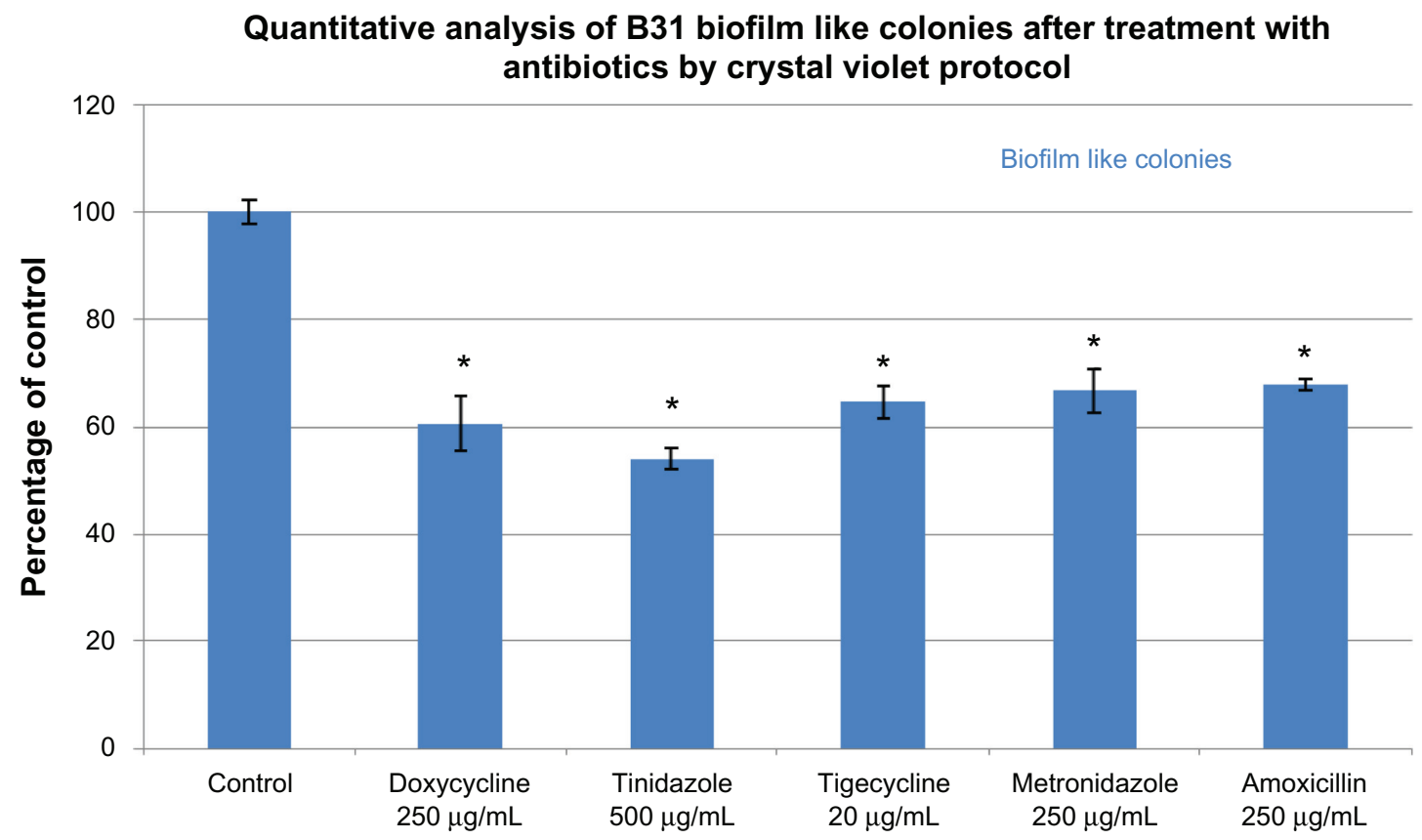

B

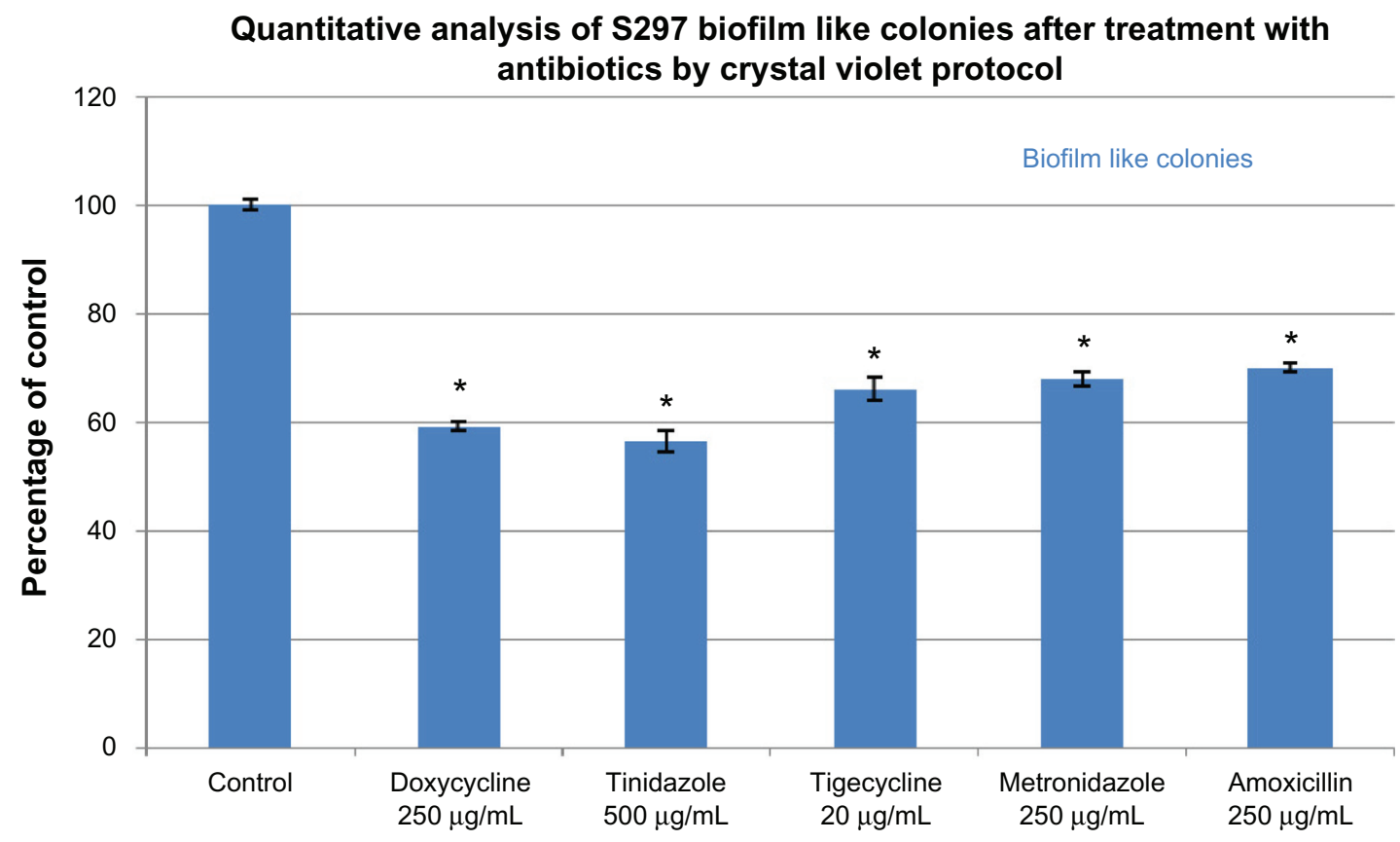

Figure 4A Evaluation of biofilm-like colonies of B. burgdorferi. Quantitative analysis of biofilm-like colonies of strain B3I (top panel) and strain S297 (bottom panel) measured by crystal violet staining technique.

that mimic tissue-based in-vivo conditions in order to optimize the antibiotic treatment of Lyme disease.

\section{Acknowledgments}

The authors thank Drs Willy Burgdorfer, Robert Lane, Alan MacDonald, Christian Melander, and Yi Zhang for helpful discussion. We are also grateful to Lorraine Johnson, Kris Newby, and Pam Weintraub for their input. This work was supported by grants from the California Lyme Disease Association, Turn the Corner Foundation and the University of New Haven. Ethical approval was not required for this research study. 

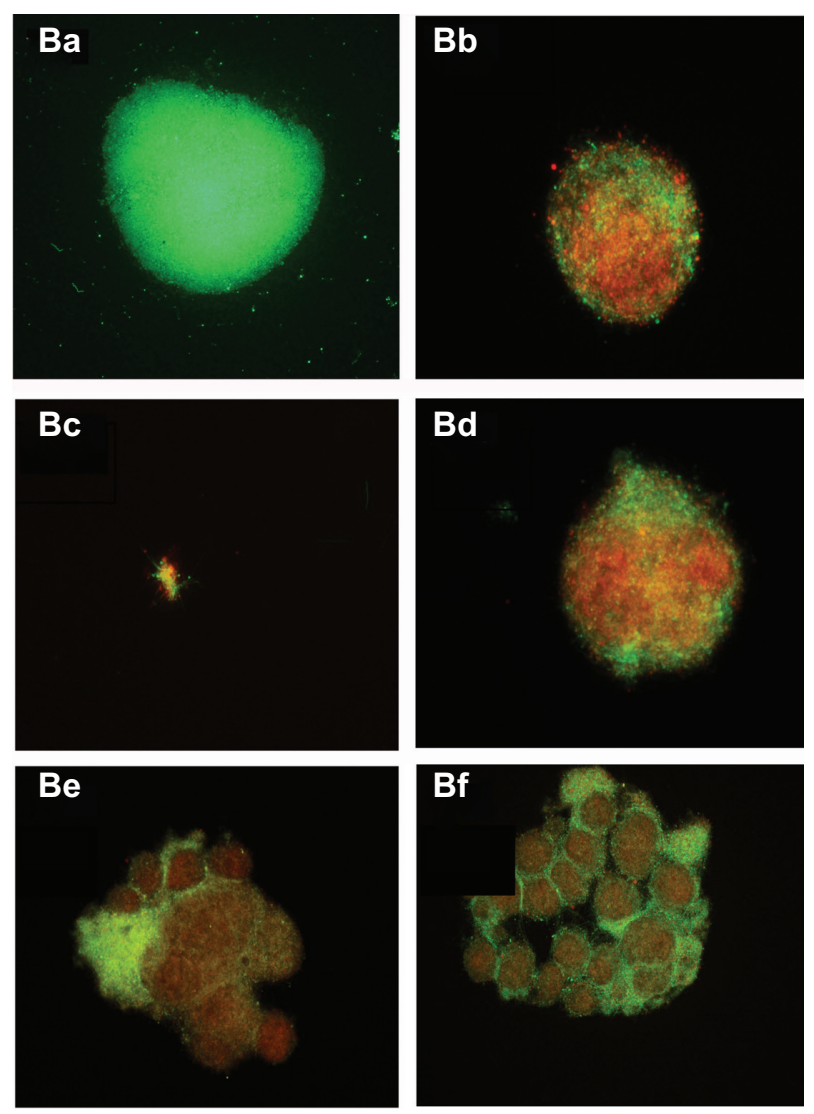

Figure 4B Evaluation of biofilm-like colonies of B. burgdorferi. Qualitative analysis of biofilm-like colonies of strain B3I measured by fluorescent microscopy using SYTO ${ }^{\circledR 9}$ green-fluorescent stain (live organisms) and propidium iodide red-fluorescent stain (dead organisms): (Ba) Control; (Bb) Doxycycline; (Bc) Tinidazole; (Bd) Tigecycline; (Be) Metronidazole; (Bf) Amoxicillin.

Note: All images taken at $40 \times$ magnification.

\section{Disclosure}

RBS serves without compensation on the medical advisory panel for QMedRx Inc. He has no financial ties to the company. The other coauthors have no potential conflicts of interest to declare.

\section{References}

1. Burgdorfer W, Barbour AG, Hayes SF, Benach JL, Grunwaldt E, Davis JP. Lyme disease-a tick-borne spirochetosis? Science. 1982;216:1317-1319.

2. Stricker RB, Johnson L. Lyme disease: the next decade. Infect Drug Resist. 2011;4:1-9.

3. Van Dam AP, Kuiper H, Vos K, et al. Different genospecies of Borrelia burgdorferi are associated with distinct clinical manifestations of Lyme borreliosis. Clin Infect Dis. 1993;17:708-717.

4. Stricker RB, Johnson L. Lyme disease diagnosis and treatment: lessons from the AIDS epidemic. Minerva Med. 2010;101:419-425.

5. Bacon RM, Kugeler KJ, Mead PS; for the Centers for Disease Control and Prevention (CDC). Surveillance for Lyme disease - United States, 1992-2006. MMWR Surveill Summ. 2008;57:1-9.

6. Meek JI, Roberts CL, Smith EV, Cartter ML. Underreporting of Lyme disease by Connecticut physicians, 1992. J Public Health Manag Pract. 1996;2:61-65.

7. Boltri JM, Hash RB, Vogel RL. Patterns of Lyme disease diagnosis and treatment by family physicians in a southeastern state. $J$ Community Health. 2002;27:395-402.
8. Liegner KB, Shapiro JR, Ramsay D, Halperin AJ, Hogrefe W, Kong L. Recurrent erythema migrans despite extended antibiotic treatment with minocycline in a patient with persisting Borrelia burgdorferi infection. J Am Acad Dermatol. 1993;28(2 Pt 2):312-314.

9. Donta ST. Tetracycline therapy for chronic Lyme disease. Clin Infect Dis. 1997;25(Suppl 1):S52-S56.

10. Donta ST. Macrolide therapy of chronic Lyme disease. Med Sci Monit. 2003;9:136-142.

11. Krause PJ, Foley DT, Burke GS, Christianson D, Closter L, Spielman A. Reinfection and relapse in early Lyme disease. Am Trop Med Hyg. 2006;75:1090-1094.

12. Hodzic, E, Feng S, Holden K, Freet K, Barthold SW. Persistence of Borrelia burgdorferi following antibiotic treatment in mice. Antimicrob Agents Chemother. 2008;52:1728-1736.

13. Straubinger RK. PCR-based quantification of Borrelia burgdorferi organisms in canine tissues over a 500-day post-infection period. J Clin Microbiol. 2000;38:2191-2199.

14. Oksi J, Marjamaki M, Nikoskelainen J, Viljanen MK. Borrelia burgdorferi detected by culture and PCR in clinical relapse of disseminated Lyme borreliosis. Ann Med. 1999;31:225-232.

15. Ge Y, Li C, Corum L, Slaughter CA, Charon NW. Structure and expression of the FlaA periplasmic flagellar protein of Borrelia burgdorferi. J Bacteriol. 1998;180:2418-2425.

16. Sal MS, Li C, Motalab MA, Shibata S, Aizawa S, Charon NW. Borrelia burgdorferi uniquely regulates its motility genes and has an intricate flagellar hook-basal body structure. J Bacteriol. 2008;190:1912-1921.

17. Livengood JA, Gilmore RJ. Invasion of human neuronal and glial cells by an infectious strain of Borrelia burgdorferi. Microbes Infect. 2006; 8:2832-2840.

18. Wu J, Weening EH, Faske JB, Höök M, Skare JT. Invasion of eukaryotic cells by Borrelia burgdorferi requires $\beta 1$ integrins and Src kinase activity. Infect Immun. 2011;79:1338-1348.

19. Alban PS, Johnson PW, Nelson DR. Serum-starvation-induced changes in protein synthesis and morphology of Borrelia burgdorferi. Microbiology. 2000;146:119-127.

20. Ramamoorthy R, Scholl-Meeker D. Borrelia burgdorferi proteins whose expression is similarly affected by culture temperature and $\mathrm{pH}$. Infect Immun. 2001;69:2739-2742.

21. Murgia R, Cinco M. Induction of cystic forms by different stress conditions in Borrelia burgdorferi. APMIS. 2004;112:57-62.

22. Brorson Ø, Brorson SH. In vitro conversion of Borrelia burgdorferi to cystic forms in spinal fluid, and transformation to mobile spirochetes by incubation in BSK-H medium. Infection. 1998;26:144-150.

23. Brorson Ø, Brorson SH. Transformation of cystic forms of Borrelia burgdorferi to normal mobile spirochetes. Infection. 1997;25:240-246.

24. Al-Robaiy S, Dihazi H, Kacza J, et al. Metamorphosis of Borrelia burgdorferi organisms - RNA, lipid and protein composition in context with the spirochetes' shape. J Basic Microbiol. 2010;50(Suppl 1): S5-S17.

25. Kersten A, Poitschek C, Rauch S, Aberer E. Effects of penicillin, ceftriaxone, and doxycycline on morphology of Borrelia burgdorferi. Antimicrob Agents Chemother. 1995;39:1127-1133.

26. Sapi E, MacDonald A. Biofilms of Borrelia burgdorferi in chronic cutaneous borreliosis. Am J Clin Pathol. 2008;129:988-989.

27. Eisendle K, Müller H, Zelger B. Biofilms of Borrelia burgdorferi in chronic cutaneous borreliosis. Am J Clin Pathol. 2008;129:989-990.

28. Jeffereson KK. What drives bacteria to form a biofilm? FEMS Microbiol Lett. 2004;52:917-924.

29. Stewart P, Costerton JW. Antibiotic resistance of bacteria in biofilms. Lancet. 2001;358:135-138.

30. Costerton JW, Stewart PS, Greenburg EP. Bacterial biofilms, a common cause of persistent infections. Science. 1999;284:1318-1322.

31. Hunfeld KP, Kraiczy P, Kekoukh E, Schäfer V, Brade V. Standardized in vitro susceptibility testing of Borrelia burgdorferi against well-known and newly developed antimicrobial agents - possible implications for new therapeutic approaches to Lyme disease. Int J Med Microbiol. 2002;291(Suppl 33):125-137. 
32. Dever LL, Jorgensen JH, Barbour AG. In vitro antimicrobial susceptibility testing of Borrelia burgdorferi: a micro-dilution MIC method and time-kill studies. J Clin Microbiol. 1992;30:2692-2697.

33. Boerner J, Failing K, Wittenbrink MM. In vitro antimicrobial susceptibility testing of Borrelia burgdorferi: influence of test conditions on minimal inhibitory concentration (MIC) values. Zentralbl Bakteriol. 1995;283:49-60.

34. Siegemund M, van Bommel J, Ince C. Assessment of regional tissue oxygenation. Intensive Care Med. 1999;25:1044-1060.

35. Venkatesh B, Morgan TJ, Lipman J. Subcutaneous oxygen tensions provide similar information to ileal luminal CO2 tensions in an animal model of hemorrhagic shock. Intensive Care Med. 2000;26:592-600.

36. Ruzic-Sabljic E, Strle F. Comparison of growth of Borrelia afzelii, B. garinii, and B. burgdorferi sensu stricto in MKP and BSK-II medium. Int J Med Microbiol. 2004;294:407-412.

37. Wang G, Iyer R, Bittker S, et al. Variations in Barbour-Stoenner-Kelly culture medium modulate infectivity and pathogenicity of Borrelia burgdorferi clinical isolates. Infect Immun. 2004;72:6702-6706.

38. Brorson $\varnothing$, Brorson SH. An in vitro study of the susceptibility of mobile and cystic forms of Borrelia burgdorferi to tinidazole. International Microbiol. 2004;7:139-142.

39. Brorson Ø, Brorson SH, Scythes J, MacAllister J, Wier A, Margulis L. Destruction of spirochete Borrelia burgdorferi round-body propagules (RBs) by the antibiotic tigecycline. Proc Natl Acad Sci U SA. 2009;106: 18656-18661.
40. Mazzei T, Mini E, Novelli A, Periti P. Chemistry and mode of action of macrolides. J Antimicrob Chemother. 1993;31(Suppl C):1-9.

41. Chopra I, Roberts M. Tetracycline antibiotics: mode of action, applications, molecular biology, and epidemiology of bacterial resistance. Microbiol Mol Biol Rev. 2001;65:232-260.

42. Tenson T, Lovmar M, Ehrenberg M. The mechanism of action of macrolides, lincosamides and streptogramin B reveals the nascent peptide exit path in the ribosome. J Mol Biol. 2003;330:1005-1014.

43. Kasbekar N, Acharya PS. Telithromycin: the first ketolide for the treatment of respiratory infections. Am J Health Syst Pharm. 2005; 62:905-916.

44. Goldman P. The development of 5-nitroimidazoles for the treatment and prophylaxis of anaerobic bacterial infections. JAntimicrob Chemother. 1982;10:23-33.

45. Slover CM, Rodvold KA, Danziger LH. Tigecycline: a novel broadspectrum antimicrobial. Ann Pharmacother. 2007;41:965-972.

46. Barthold SW, Hodzic E, Imai DM, Feng S, Yang X, Luft BJ. Ineffectiveness of tigecycline against persistent Borrelia burgdorferi. Antimicrob Agents Chemother. 2010;54:643-651.
Infection and Drug Resistance

\section{Publish your work in this journal}

Infection and Drug Resistance is an international, peer-reviewed openaccess journal that focuses on the optimal treatment of infection (bacterial, fungal and viral) and the development and institution of preventive strategies to minimize the development and spread of resistance. The journal is specifically concerned with the epidemiology of antibiotic

\section{Dovepress}

resistance and the mechanisms of resistance development and diffusion in both hospitals and the community. The manuscript management system is completely online and includes a very quick and fair peerreview system, which is all easy to use. Visit http://www.dovepress.com/ testimonials.php to read real quotes from published authors. 